\title{
Carbon monoxide total column retrievals from TROPOMI shortwave infrared measurements
}

\author{
Jochen Landgraf $^{1}$, Joost aan de Brugh ${ }^{1}$, Remco Scheepmaker ${ }^{1}$, Tobias Borsdorff ${ }^{1}$, Haili Hu ${ }^{1}$, Sander Houweling $^{1,3}$, \\ Andre Butz ${ }^{2}$, Ilse Aben ${ }^{1}$, and Otto Hasekamp ${ }^{1}$ \\ ${ }^{1}$ SRON Netherlands Institute for Space Research, Utrecht, the Netherlands \\ ${ }^{2}$ Deutsches Zentrum für Luft- und Raumfahrt e.V. (DLR), Institut für Physik der Atmosphäre, \\ Oberpfaffenhofen, Germany \\ ${ }^{3}$ Institute for Marine and Atmospheric Research Utrecht, Utrecht, the Netherlands \\ Correspondence to: Jochen Landgraf (j.landgraf@sron.nl)
}

Received: 2 April 2016 - Published in Atmos. Meas. Tech. Discuss.: 25 May 2016

Revised: 1 September 2016 - Accepted: 14 September 2016 - Published: 7 October 2016

\begin{abstract}
The Tropospheric Monitoring Instrument (TROPOMI) spectrometer is the single payload of the Copernicus Sentinel 5 Precursor (S5P) mission. It measures Earth radiance spectra in the shortwave infrared spectral range around $2.3 \mu \mathrm{m}$ with a dedicated instrument module. These measurements provide carbon monoxide $(\mathrm{CO})$ total column densities over land, which for clear sky conditions are highly sensitive to the tropospheric boundary layer. For cloudy atmospheres over land and ocean, the column sensitivity changes according to the light path through the atmosphere. In this study, we present the physics-based operational S5P algorithm to infer atmospheric CO columns satisfying the envisaged accuracy $(<15 \%)$ and precision $(<10 \%)$ both for clear sky and cloudy observations with low cloud height. Here, methane absorption in the $2.3 \mu \mathrm{m}$ range is combined with methane abundances from a global chemical transport model to infer information on atmospheric scattering. For efficient processing, we deploy a linearized two-stream radiative transfer model as forward model and a profile scaling approach to adjust the $\mathrm{CO}$ abundance in the inversion. Based on generic measurement ensembles, including clear sky and cloudy observations, we estimated the $\mathrm{CO}$ retrieval precision to be $\leq 11 \%$ for surface albedo $\geq 0.03$ and solar zenith angle $\leq 70^{\circ}$. CO biases of $\leq 3 \%$ are introduced by inaccuracies in the methane a priori knowledge. For strongly enhanced $\mathrm{CO}$ concentrations in the tropospheric boundary layer and for cloudy conditions, $\mathrm{CO}$ errors in the order of $8 \%$ can be introduced by the retrieval of cloud parameters of our algorithm. Moreover,
\end{abstract}

we estimated the effect of a distorted spectral instrument response due to the inhomogeneous illumination of the instrument entrance slit in the flight direction to be $<2 \%$ with pseudo-random characteristics when averaging over space and time. Finally, the $\mathrm{CO}$ data exploitation is demonstrated for a TROPOMI orbit of simulated shortwave infrared measurements. Overall, the study demonstrates that for an instrument that performs in compliance with the pre-flight specifications, the $\mathrm{CO}$ product will meet the required product performance well.

\section{Introduction}

Measurements of the atmospheric carbon monoxide (CO) abundance are needed with temporal continuity and global coverage to improve our understanding of tropospheric chemistry and long-range transport (Levy, 1971; Logan et al., 1981; Shindell et al., 2006; Edwards et al., 2004). Vertically integrated total column densities of $\mathrm{CO}$ can be inferred from satellite measurements of Earth-reflected sunlight in the $2.3 \mu \mathrm{m}$ spectral range of the shortwave infrared (SWIR) part of the solar spectrum. The retrievals deliver sensitivity to the tropospheric boundary layer using the first overtone 2-0 absorption band of CO between $2305 \mathrm{~nm}$ and $2385 \mathrm{~nm}$. Under clear sky conditions, this spectral range is subject to little atmospheric scattering, and most of the measured light is thus reflected by the Earth's surface. Therefore, SWIR measurements are sensitive to the vertically integrated total amount 
of $\mathrm{CO}$, including the contribution of the planetary boundary layer. This makes the SWIR spectral range particularly suitable for detecting surface sources of $\mathrm{CO}$ from space.

With the launch of SCIAMACHY (Scanning Imaging Absorption Spectrometer for Atmospheric Chartography, Bovensmann et al., 1999) in the year 2002 on ESA's Envisat satellite, global CO SWIR measurements are available for the years 2003-2012 (Borsdorff et al., 2016). The MOPITT (Measurements of Pollution in the Troposphere; Drummond and Mand, 1996) instrument, launched by NASA on board the Terra satellite in 1999, measures atmospheric CO abundance from the SWIR (Deeter et al., 2009) in addition to thermal infrared observations in the $4.7 \mu \mathrm{m}$ CO fundamental band. To ensure continuity of SWIR CO measurements in the future, new space-borne instrumentation is required. In this respect, the Sentinel 5 Precursor mission (S5P; Veefkind, 2012), to be launched at the end of 2016, will extend these unique long-term global $\mathrm{CO}$ data sets using measurements of the same spectral range, and so bridges the data gap to the Sentinel 5 (S5) mission scheduled for launch in the year 2020.

The S5P satellite, with a designed 7-year lifetime, will fly in a sun-synchronous orbit at $824 \mathrm{~km}$ altitude with an inclination of $98.7^{\circ}$. It has the Tropospheric Monitoring Instrument (TROPOMI) as a single payload, which is a push-broom imaging spectrometer with a swath of $2600 \mathrm{~km}$. TROPOMI will provide daily global coverage with a high spatial resolution of $7 \times 7 \mathrm{~km}^{2}$ at subsatellite point. It comprises two spectrometer modules, the first covering the ultraviolet, visible and near-infrared spectral ranges and the second covering the shortwave infrared spectral range $2305-2385 \mathrm{~nm}$ with a spectral resolution of $0.25 \mathrm{~nm}$ and a spectral sampling distance of $0.1 \mathrm{~nm}$. A typical SWIR transmission spectrum is illustrated in the top panel of Fig. 1. It shows the total transmittance of solar light along its path from the sun reflected at the surface towards the satellite. The transmittance is simulated using the Beer's extinction law. In this spectral range, the relevant absorbing species are $\mathrm{H}_{2} \mathrm{O}$ its isotopologue $\mathrm{HDO}$, $\mathrm{CO}$ and $\mathrm{CH}_{4}$, with the optical depth of $\mathrm{CO}$ generally much smaller than those of $\mathrm{H}_{2} \mathrm{O}$ and $\mathrm{CH}_{4}$. The SWIR spectrometer is designed for a minimum signal-to-noise ratio of 100-120 in the continuum of the spectrum over land surfaces. Over the oceans under clear sky conditions, the SWIR signal is too low due to the dark sea surface. So CO data processing is only possible for cloudy ocean observations. Due to these unique mission characteristics, TROPOMI will allow for unprecedented observations of $\mathrm{CO}$ total column abundances to quantify its sources and sinks.

The Copernicus ground segment generates the $\mathrm{CO}$ total column data as part of the near-real-time and offline data stream. Near-real-time products will be delivered within $3 \mathrm{~h}$ after data acquisition. The full data quality will be achieved only for the offline data products, which are expected to be available within a few days after acquisition. For both data deliveries, an efficient $\mathrm{CO}$ retrieval algorithm is required.

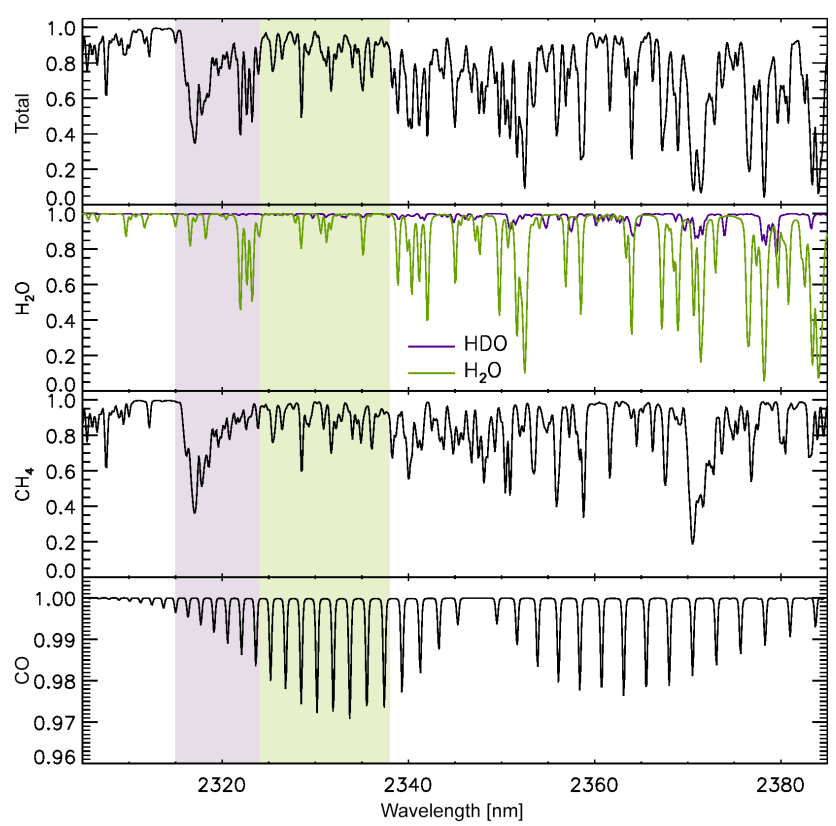

Figure 1. SWIR spectral transmittance along the light path of the solar beam reflected at the Earth surface into the instrument's viewing direction. Simulations are performed for viewing zenith angle $\mathrm{VZA}=0^{\circ}$, and a solar zenith angle $\mathrm{SZA}=30^{\circ}$, and by assuming a US standard atmospheric profile. From top to bottom, the figure shows the total transmittance, the individual transmittances due to $\mathrm{H}_{2} \mathrm{O}$ (green line), $\mathrm{HDO}$ (purple line), $\mathrm{CH}_{4}$ and $\mathrm{CO}$, respectively. The purple region indicates the spectral range $2315-2324 \mathrm{~nm}$ that is used for cloud filtering, whereas the green area highlights the adjacent spectral range $2324-2338 \mathrm{~nm}$, which is used to infer CO total columns from the measurements. Note the different $y$ axis scale for the $\mathrm{CO}$ transmittance.

Several fast algorithms were used to infer $\mathrm{CO}$ columns from SCIAMACHY SWIR measurements, including the Weighting Function Modified Differential Optical Absorption Spectroscopy approach (WFM-DOAS, Buchwitz et al. (2007) and references therein), the Iterative Maximum A Posteriori approach (IMAP, Frankenberg et al., 2005), the Beer Infrared Retrieval Algorithm (BIRRA, Gimeno García et al., 2011), and the Iterative Maximum Likelihood Method approach (IMLM, Gloudemans et al., 2009, and references therein). These algorithms retrieve vertically integrated CO column density over land and above clouds over oceans. Buchwitz et al. (2006) and Gloudemans et al. (2009) use a priori methane information to characterize the light path through the atmosphere.

Based on these concepts, Vidot et al. (2012) proposed the Shortwave Infrared Carbon Monoxide Retrieval (SICOR) algorithm for the processing of CO total columns from S5P and S5 shortwave infrared measurements. The algorithm describes the effect of clouds on the radiation field by an elevated Lambertian reflector of a fixed albedo, adjusting the elevation height and the cloud coverage of the observed scene. 
This approach accounts well for the effect of optically thick water clouds on the $\mathrm{CO}$ retrieval with biases $<3 \%$, but introduces larger biases for an elevated aerosol layer above bright surfaces as well as optically thin cirrus clouds in the upper troposphere. Here the photon path length is significantly enhanced due to photon trapping between the aerosol or cirrus layer and the surface, which represents a clear drawback of the approach. The study at hand analyzes recent advancements in developing the SICOR algorithm, amongst others using a linearized two-stream radiative model to account for atmospheric scattering. Here we give particular attention to the TROPOMI specific instrument aspects, and we discuss the expected algorithm performance in the context of the operational data processing of the S5P mission.

The paper is structured as follows: Sect. 2 describes the retrieval method including the basic features of the forward model. More details on the linearized two-stream radiative transfer model are given in Appendix A. In Sect. 3, we present the uncertainty analysis of the $\mathrm{CO}$ product with respect to atmospheric and critical instrument parameters based on generic measurement scenarios, whereas Sect. 4 illustrates the TROPOMI CO data product for a simulated level-1b orbit ensemble. Finally, Sect. 5 concludes the paper.

\section{Retrieval algorithm}

The TROPOMI CO retrieval algorithm infers information on the total amount of $\mathrm{CO}$ from SWIR measurements, focussing on clear-sky observations over land and cloudy observations over land and ocean in the presence of low-altitude liquid water clouds. Figure 2 summarizes the SICOR algorithm. The dynamic input includes S5P level 1b data, which comprises solar irradiance and Earth radiance spectra in the spectral range $2315-2338 \mathrm{~nm}$, forecast (FCST) data on atmospheric pressure $p$, temperature $T$ and specific humidity $q$, the terrain's elevation from a digital elevation model (DEM) and a priori information on the $\mathrm{CO}$ and $\mathrm{CH}_{4}$ vertical distribution of the observed atmosphere coming from a chemical transport model (CTM). These input data and their required accuracy will be discussed in more detail in Sect. 3 . The first processing step screens the data to filter out observations with high and optically thick clouds. Subsequently, we utilize a physics-based retrieval approach to infer CO columns from SWIR measurements together with the atmospheric $\mathrm{H}_{2} \mathrm{O}$ abundances, surface albedo and a spectral calibration of the measurement spectrum. The spectral absorption by methane is used to infer information on atmospheric scattering by clouds and aerosols. Finally, the algorithm has the retrieved CO total column, the corresponding column averaging kernel and an estimate of the random error component as output. The theoretical baseline of our algorithm is described in detail in the following.

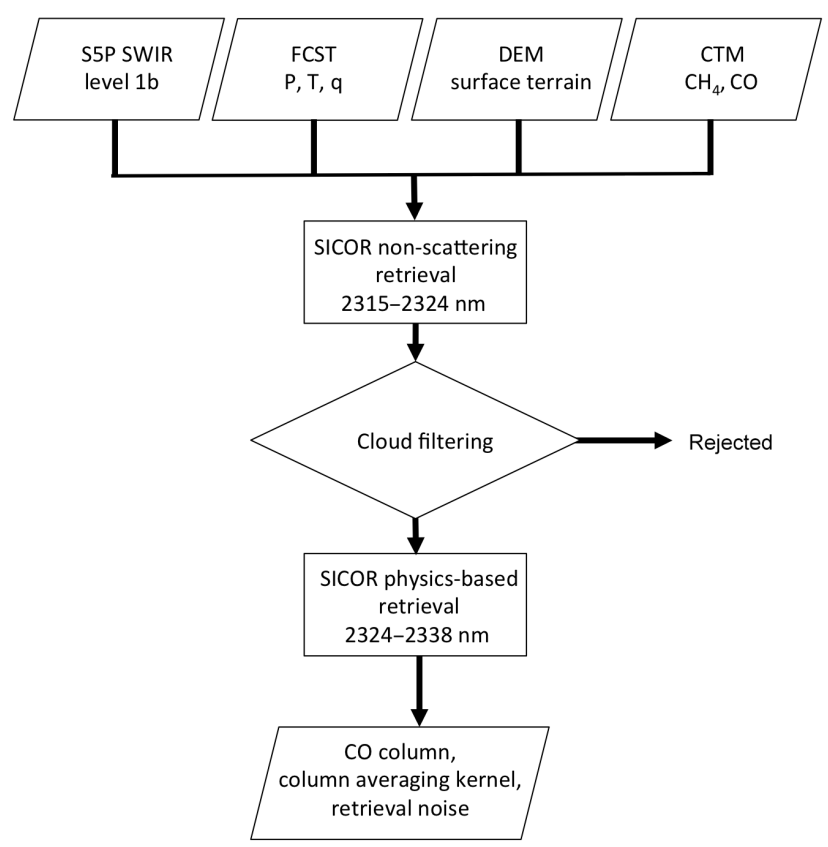

Figure 2. Flowchart of the SICOR algorithm.

\subsection{Cloud filtering}

To detect the presence of high, optically thick clouds, we infer the vertically integrated amount of methane from measurements between 2315 and $2324 \mathrm{~nm}$ (see Fig. 1) using a radiative transfer model that neglects atmospheric scattering. The difference $\Delta \mathrm{CH}_{4}$ between the retrieved $\mathrm{CH}_{4}$ column and a priori methane information coming from a chemical transport model indicates light path modification, either shortening or enhancement in comparison with the direct light path from the sun to the spectrometer via reflection at the Earth surface, due to atmospheric scattering by clouds and aerosols. Here the net effect depends on the scattering properties such as scattering height and optical depth, surface reflection and solar and observation geometry (e.g., Aben et al., 2007). If the difference exceeds a certain threshold, observations are rejected. The non-scattering retrieval algorithm uses a standard least squares approach to infer the total column of $\mathrm{CH}_{4}, \mathrm{CO}, \mathrm{H}_{2} \mathrm{O}$ and $\mathrm{HDO}$ together with a surface albedo $A_{\mathrm{s}}$, its linear dependence on wavelength and a spectral offset. It is described in more detail by Scheepmaker et al. (2016).

Figure 3 shows the probability density function (PDF) and its cumulative distribution (CPDF) of the difference $\Delta \mathrm{CH}_{4}$ between a non-scattering $\mathrm{CH}_{4}$ retrieval from observations of Greenhouse Gases Observing Satellite (GOSAT, Kuze et al., 2009) at the $1.6 \mu \mathrm{m}$ band of the year 2010 over land and ocean and collocated $\mathrm{CH}_{4}$ columns from TM5 model simulations after optimization using surface measurements (Houweling et al., 2014), relative to the model results. The maximum of the ocean and land PDF is at small differences $\Delta \mathrm{CH}_{4}$, indicating a large number of scenes that are affected 

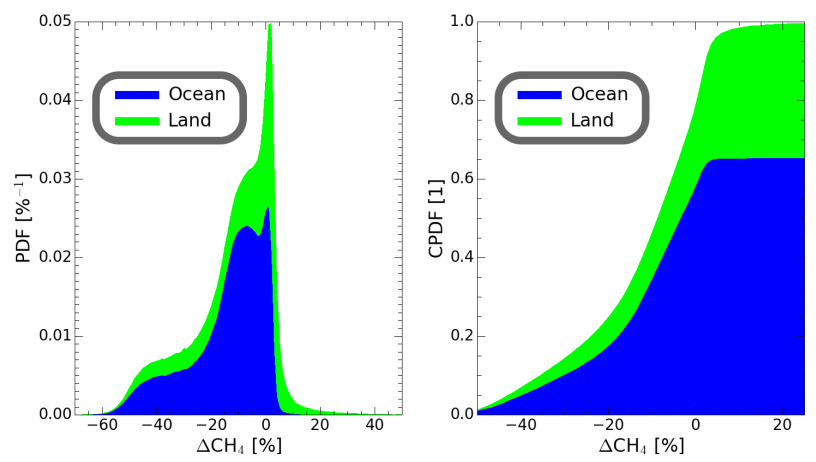

Figure 3. Probability density function (left panel) and cumulative probability density function (right panel) of the difference $\Delta \mathrm{CH}_{4}$ of 1 year of GOSAT observations (2010) minus the corresponding TM5 model simulations. The figure differentiates between the contribution of ocean and land pixels (blue and green areas).

only little by clouds. For about $80 \%$ of all observations, the methane abundance is underestimated by the non-scattering retrieval due to the presence of optically thick clouds. Here, the ocean PDF shows a relatively high probability for $\Delta \mathrm{CH}_{4}$ between -20 and $-5 \%$ due to the presence of low stratiform clouds over ocean. For land pixels, this type of cloudiness occurs less frequently. Finally, $20 \%$ of all cases show an overestimation of methane by the non-scattering retrieval, indicating an effective pathlength enhancement. Although the effect of light path shorting and enhancement may depend on wavelength, because of the spectral dependence of the surface albedo and the optical properties of the atmosphere, the GOSAT PDF of $\Delta \mathrm{CH}_{4}$ provides a first estimate of the corresponding TROPOMI PDF of methane retrievals at $2.3 \mu \mathrm{m}$. As a baseline for our data selection, we accept all observations with $\left|\Delta \mathrm{CH}_{4}\right| \leq 25 \%$.

\subsection{Forward Model}

The physics-based retrieval of $\mathrm{CO}$ requires a forward model $\boldsymbol{F}$ that describes the measurement as a function of the atmospheric state including an appropriate description of atmospheric scattering,

$\boldsymbol{y}=\boldsymbol{F}(x, b)+\boldsymbol{e}_{\boldsymbol{y}}$.

Here, vector $\boldsymbol{y}$ has the spectral measurements between 2324 and $2338 \mathrm{~nm}$ as its components (see Fig. 1), state vector $\boldsymbol{x}$ represents the parameters to be retrieved, $\boldsymbol{b}$ describes parameters other than the state vector that influences the measurement but are not adjusted by the retrieval and $\boldsymbol{e}_{\boldsymbol{y}}$ is the measurement error. The fit window compromises about optimal CO sensitivity, little interference with water vapor and methane absorptions and small forward model errors due to the assumed cloud model. Moreover, the forward model is nonlinear in the state vector $\boldsymbol{x}$. Therefore, the inversion problem is solved iteratively employing the Gauss-Newton method, where for each iteration step the forward model is linearized by a Taylor expansion around the solution of the previous iteration $\boldsymbol{x}_{o}$ :

$\boldsymbol{F}(\boldsymbol{x}, \boldsymbol{b})=\boldsymbol{F}\left(\boldsymbol{x}_{0}, \boldsymbol{b}\right)+\frac{\partial \boldsymbol{F}}{\partial \boldsymbol{x}}\left(\boldsymbol{x}_{0}, \boldsymbol{b}\right)\left\{\boldsymbol{x}-\boldsymbol{x}_{0}\right\}+\mathcal{O}\left(\left(\boldsymbol{x}-\boldsymbol{x}_{0}\right)^{2}\right)$.

$\mathcal{O}\left(x^{2}\right)$ indicates second and higher order contributions of the expansion.

The forward model $\boldsymbol{F}$ simulates the Earth radiance measurement by a spectral convolution of the top-of-modelatmosphere radiance $I^{\mathrm{TOA}}$ with the instrument spectral response function:

$F_{i}=s_{i} \cdot I^{\mathrm{TOA}}=\int s_{i}(\lambda) I^{\mathrm{TOA}}(\lambda) \mathrm{d} \lambda$.

Here, $s_{i}$ describes the spectral instrument response of spectral pixel $i$ with the assigned wavelength $\lambda_{i}$, and $I^{\mathrm{TOA}}(\lambda)$ is simulated by a line-by-line radiative transfer model on a fine internal spectral grid. This model requires a solar irradiance spectrum on the internal spectral grid as input, which is inferred from the daily solar measurements of TROPOMI using the deconvolution approach by van Deelen et al. (2007) and Wassmann et al. (2015).

State-of-the-art radiative transfer models account for multiple scattering in multiple propagation directions (streams) including the polarization of light. For our application, the computational effort of such simulations is far too large, and thus approximation methods are required to accelerate the forward model simulations. For this reason, we ignore atmospheric Rayleigh scattering, which contributes less than $0.15 \%$ to the total signal (Gloudemans et al., 2008), and use a numerically efficient two-stream scalar radiative transfer model to describe scattering by clouds and aerosols. The employed two-stream solver (2S-LINTRAN) calculates the amount of singly scattered light, whereas the diffuse radiation is approximated by two propagation directions of the radiance field, one upward and one downward. It is similar to the model by Spurr and Natraj (2011), and the numerical implementation used for the S5P CO column retrieval is described in more detail in Appendix A.

In the forward model, clouds and aerosols are represented by a scattering layer with a triangular height profile in optical depth with a center height $z_{\text {scat }}$ and a fixed full width at half maximum of $2.5 \mathrm{~km}$. Within the scattering layer we assume a constant single scattering albedo and scattering phase function. In this case, we can optimize the numerical efficiency of the two-stream solver using an aggregated vertical grid. In a first step, we calculate absorption optical depth on a $1 \mathrm{~km}$ vertical grid accounting for the pressure and temperature dependence of atmospheric absorption, and subsequently we combine the atmosphere layers above and below the scattering layer to one layer each by integrating the optical depth. This significantly reduces the number of vertical layers in the radiative transfer simulation (typically to less than 10), depending on the number of internal layers that are used to 
resolve the height profile of the scattering layer. Finally, the optical properties of the scattering layer have to be known a priori and we chose a spectrally constant single-scattering albedo $\omega=0.9$ and an asymmetry parameter of the scattering phase function $g=0.7$. Moreover, we use a simplified wavelength dependence of the extinction optical thickness of the scattering layer:

$\tau(\lambda)=\tau\left(\lambda_{0}\right)\left(\frac{\lambda}{\lambda_{0}}\right)^{-\alpha}$,

where the reference wavelength $\lambda_{0}=2331 \mathrm{~nm}$ is chosen at the center of our fitting window and $\alpha=1.0$ is the Angström parameter. These a priori assumptions on the optical parameters of clouds and aerosols will be justified by the performance analysis in Sect. 4, which is based on measurement simulations of one TROPOMI orbit for a realistic variation of cloud and aerosol properties.

In spite of the efficiency of the radiative transfer solver, the numerical cost of the forward model has to be reduced further for operational data processing. Therefore, we precalculate the molecular absorption cross sections $\sigma$ of $\mathrm{CH}_{4}$, $\mathrm{H}_{2} \mathrm{O}$, HDO and $\mathrm{CO}$ as a function of pressure, temperature and for a spectral sampling distance of $5 \times 10^{-3} \mathrm{~cm}^{-1}$ from spectroscopic databases (Rothman et al., 2009; Predoi-Cross et al., 2006 for $\mathrm{CO}$ and $\mathrm{CH}_{4}$ respectively, and Scheepmaker et al., 2013 for water vapor and its isotopologues). From this data set, we derive cross sections by bilinear interpolation of the pressure and temperature for each individual retrieval layer followed by the calculation of effective cross sections $\sigma_{\text {eff }}$ per species on a coarser spectral sampling $k_{i}$ by the generalized mean

$\sigma_{\text {eff }}\left(k_{i}\right)=\sqrt[m]{\frac{\int T_{i}(k) \sigma^{m}(k) \mathrm{d} k}{\int T_{i}(k) \mathrm{d} k}}$,

with a spectral sampling of $3 \times 10^{-2} \mathrm{~cm}^{-1}$. Here, $k$ represents wavenumber and $T_{i}(k)$ is a normalized symmetric triangular weighting function between spectral samplings $k_{i-1}$ and $k_{i+1}$ with a peak at $k_{i}$. For $m=1$, Eq. (5) describes the arithmetic mean, which introduces significant forward model errors in the retrieval for the envisaged spectral sampling. We performed retrieval experiments for different values of $m$, where we achieved most accurate radiance simulations with $\mathrm{CO}$ retrieval biases $<1 \%$ under clear sky conditions for $m=0.85$. Overall, the use of the effective cross sections speeds up the forward model simulations by a factor of 6 compared to line-by-line calculations on the spectral grid of the original spectroscopic database.

\subsection{Inversion}

The SWIR measurements are sensitive to the total amount of $\mathrm{CO}$ along the path of the measured light. For clear sky atmospheres and within the bounds of the measurement error, only the total column of $\mathrm{CO}$ can be inferred from the measurement (Borsdorff et al., 2016), and no information is obtained about the relative vertical distribution of CO. In the presence of clouds, the measurement loses sensitivity to the amount of $\mathrm{CO}$ below the cloud. To properly account for this, a regularized $\mathrm{CO}$ profile retrieval is required that accounts for the different sensitivity of the measurement to $\mathrm{CO}$ at different altitudes. For this purpose, we employ the Tikhonov regularization technique of first order (Phillips, 1962; Tikhonov, 1963) embedded in the Gauss-Newton iteration scheme. For each iteration step, the least square solution $\hat{\boldsymbol{x}}$ is given by

$\hat{\boldsymbol{x}}=\min _{\boldsymbol{x}}\left\{\left\|\mathbf{S}_{\boldsymbol{y}}^{-1 / 2}(\boldsymbol{F}(\boldsymbol{x})-\boldsymbol{y})\right\|^{2}+\gamma^{2}\left\|\mathbf{L}_{1} \boldsymbol{x}\right\|^{2}\right\}$.

Here, $\|\cdot\|$ describes the Euclidean norm and $\mathbf{S}_{\boldsymbol{y}}$ is the error covariance matrix of the measurement $\boldsymbol{y}$, where we assume uncorrelated measurement errors. $\gamma$ is the regularization parameter and

$\mathbf{L}_{1}=\left(\begin{array}{cccccc}-1 & 1 & 0 & \cdots & 0 & 0 \\ 0 & -1 & 1 & 0 & \cdots & 0 \\ \vdots & \vdots & \vdots & \vdots & \vdots & \vdots \\ 0 & 0 & \cdots & -1 & 1 & 0 \\ 0 & 0 & \cdots & 0 & -1 & 1\end{array}\right)$

is the first-difference operator, and so the regularization favors constant solutions and penalizes the "roughness" of $\boldsymbol{x}$. The state vector $\boldsymbol{x}$ contains the CO profile $\boldsymbol{x}_{\mathrm{CO}}$, which is expressed relative to a reference profile $\boldsymbol{\rho}^{\text {ref }}$, namely

$x_{\mathrm{CO}}=\rho / \rho^{\mathrm{ref}}$.

For the operational implementation, TM5 model fields are used to extract an adequate $\mathrm{CO}$ reference profile. Besides the relative profile of $\mathrm{CO}$, the state vector includes the water vapor column density for two isotopologues, $c_{\mathrm{H}_{2} \mathrm{O}}$ and $c_{\mathrm{HDO}}$, the surface albedo $A_{\mathrm{S}}$ and its linear dependence on wavelength $\Delta A_{\mathrm{s}}$, the effective cloud center height $z_{\text {cld }}$ and the effective cloud optical depth $\tau_{\text {cld }}$. Furthermore, a spectral shift $\Delta \lambda$ is fitted to account for spectral calibration errors of the measurement. To keep all elements of the state vector dimensionless, in addition, these entries of the state vector are normalized to a reference value. We regularize the solution in Eq. (6) such that 1 degree of freedom for signal of the retrieved $\mathrm{CO}$ profile is inferred from the measurement, bearing in mind that because of the noise the TROPOMI SWIR measurements are only sensitive to the total amount of $\mathrm{CO}$ along the path of the observed light through the atmosphere. For Eq. (6), this corresponds to a regularization parameter $\gamma \rightarrow \infty$.

Borsdorff et al. (2014) showed that the solution of this minimization problem is identical to an unregularized least squares approach:

$\hat{\boldsymbol{x}}=\min _{\boldsymbol{x}}\left\|\mathbf{S}_{\boldsymbol{y}}^{-1 / 2}(\boldsymbol{F}(\boldsymbol{x})-\boldsymbol{y})\right\|^{2}$, 
where the state vector contains the total CO column instead of the $\mathrm{CO}$ profile:

$c=\boldsymbol{C} \boldsymbol{\rho}=\int \rho(z) \mathrm{d} z$,

with the corresponding altitude integral operator $\boldsymbol{C}$. All other elements of the state vector remain the same.

The solution of this least-squares problem is

$\hat{\boldsymbol{x}}=\mathbf{G} \widetilde{\boldsymbol{y}}$

with

$\widetilde{\boldsymbol{y}}=\boldsymbol{y}-\boldsymbol{F}\left(\boldsymbol{x}_{0}\right)+\mathbf{K} \boldsymbol{x}_{0}$

and

$\mathbf{G}=\left(\mathbf{K}^{T} \mathbf{S}_{y}^{-1} \mathbf{K}\right)^{-1} \mathbf{K}^{T} \mathbf{S}_{y}^{-1}$.

Two important diagnostic tools can be calculated during the retrieval (Borsdorff et al., 2014), the error covariance matrix $\mathbf{S}_{\boldsymbol{x}}=\mathbf{G S}_{\mathbf{y}} \mathbf{G}^{T}$, which describes the effect of the measurement noise on the retrieved parameters including their correlations, and the column averaging kernel

$A^{\mathrm{col}}=\frac{\mathrm{d} \hat{c}}{\mathrm{~d} \rho^{\text {true }}}$,

which indicates the sensitivity of the retrieved column $\hat{c}$ to changes in the atmospheric $\mathrm{CO}$ profile. Here, we provide the column averaging kernel for the $\mathrm{CO}$ profile given by its partial columns of each model layer. In the linear approximation, the column averaging kernel relates the retrieved $\mathrm{CO}$ column to the true $\mathrm{CO}$ profile by

$\hat{c}=A^{\text {col }} \rho^{\text {true }}+e_{x}$,

with an error contribution $e_{x}$. Therefore, generally $\hat{c}$ does not represent an estimate of the true column and the difference

$e_{\text {null }}=\left(\boldsymbol{C}-\boldsymbol{A}^{\mathrm{col}}\right) \boldsymbol{\rho}^{\text {true }}$

is called the null-space error of the inversion. This error can be interpreted as the effect of the chosen reference profile on the retrieved CO column density (Borsdorff et al., 2014; Wassmann et al., 2015). If the reference profile to be scaled by the inversion has the correct shape, the null space error vanishes and the retrieved column represents an estimate of the true column.

Referring to Eq. (15), we characterize the retrieval accuracy for simulated measurements by the retrieval bias $b_{\mathrm{CO}}$, which is defined as the difference between the retrieved column and $\boldsymbol{A}^{\mathrm{col}} \boldsymbol{\rho}^{\text {true }}$ corrected for the retrieval noise $\boldsymbol{g}^{\mathrm{CO}} \boldsymbol{e}_{y}$ :

$b_{\mathrm{CO}}=\frac{\hat{c}-\boldsymbol{A}^{\mathrm{col}} \boldsymbol{\rho}_{\text {true }}-\boldsymbol{g}^{\mathrm{CO}} \boldsymbol{e}_{y}}{\boldsymbol{A}^{\mathrm{col}} \boldsymbol{\rho}_{\text {true }}}$.
Here $g^{\mathrm{CO}}$ is the $\mathrm{CO}$ row vector of the gain matrix in Eq. (13), and $\boldsymbol{e}_{y}$ represents the measurement noise.

The inversion described so far focused on the regularization of the ill-posed retrieval of a CO profile from SWIR measurements. The inversion remains vulnerable to other elements of the state vector to which the measurements are insensitive for certain atmospheric circumstances. For example for a scene overcast by a optically thick cloud, the measurement is insensitive to the surface albedo. On the other hand, for a clear sky observation the adjustment of the surface albedo is required but the measurement is insensitive to the height of a possible cloud layer. Hence for these circumstances, certain eigenvalues of the normal matrix $\left(\mathbf{K}^{T} \mathbf{S}_{y}^{-1} \mathbf{K}\right)$ approach zero, leading to singularities in the inversion. To overcome this, we apply Tikhonov regularization of zeroth order to the relevant elements of the state vector, namely

$\hat{\boldsymbol{x}}=\min _{\boldsymbol{x}}\left\{\left\|\mathbf{S}_{\boldsymbol{y}}^{-1 / 2}(\boldsymbol{F}(\boldsymbol{x})-\boldsymbol{y})\right\|^{2}+\gamma^{2}\|\mathbf{W} \boldsymbol{x}\|^{2}\right\}$,

where $\mathbf{W}$ is a diagonal weighting matrix, of which diagonal elements are one for all elements of the state vector related to the scattering layer and the surface albedo, i.e., $A_{\mathrm{s}}, \Delta A_{\mathrm{s}}$, $z_{\text {cld }}$ and $\tau_{\text {cld }}$, and zero otherwise.

The implementation of the SICOR inversion algorithm is based on the minimization problem (Eq. 18). It comprises the $\mathrm{CO}$ profile scaling approach as a particular regularization of the CO profile retrieval in Eq. (6). For the software implementation, we make use of the fact that its solution is identical to the least squares problem (Eq. 9), where the atmospheric abundance of $\mathrm{CO}$ abundance is adjusted through scaling of a reference profile. Finally to prevent numerical instabilities, we introduce a second regularization in Eq. (18), which mainly affects the inversion of cloud and surface parameters, and its effect on the retrieved CO column can be neglected. In this study, we have determined the regularization parameter $\gamma$ by numerical experiments (Vidot et al., 2012), which requires verification during the instrument commission phase.

Finally, the nonlinearity of the inversion is accounted by the Gauss-Newton iteration, where the degree of convergence is defined as the difference in the reduced chi-squared $\chi^{2}$ between two consecutive iteration steps, and convergence is achieved when $\left|\chi_{n}^{2}-\chi_{n-1}^{2}\right|<\epsilon$. The threshold value of $\epsilon$ can only be determined in a reliable manner using real measurements during the commissioning phase of the S5P mission. In this study, we used $\epsilon=0.5$. For clear sky observations ignoring the retrieval of a scattering layer, the Gauss-Newton scheme shows satisfying convergence properties. However, inferring cloud properties introduces significant nonlinearity issues to the retrieval. Therefore to mitigate the risk of an unstable inversion, we reduce the step sizes of the inversion during the first few iterations as described by Butz et al. (2012).

In summary, the operational S5P CO data product consists of (1) the CO vertically integrated column density $c$, (2) the 
standard deviation $\sigma$ of the $\mathrm{CO}$ retrieval noise characterized by the retrieval error covariance matrix $\mathbf{S}_{\boldsymbol{x}}$ and (3) the column averaging kernel $\boldsymbol{A}_{c}$.

\section{Sensitivity analysis}

For individual CO observations, the Sentinel 5 Precursor mission envisages a product accuracy of $\leq 15 \%$ and a precision of $\leq 10 \%$ (Veefkind, 2012). In this section, we discuss the $\mathrm{CO}$ retrieval sensitivity of our algorithm to forward model errors and a set of key atmospheric and instrument parameters, and compare these errors to the envisaged product uncertainties. To estimate the retrieval accuracy of our algorithm, we have generated synthetic measurements for generic test cases using the S-LINTRAN radiative transfer model (Schepers et al., 2015). The model is a scalar plane-parallel radiative transfer model that fully accounts for multiple elastic light scattering by clouds and air molecules and the reflection of light at the Earth surface. The optical properties of clouds are calculated using Mie theory. For ice clouds, the ray tracing model by Hess and Wiegner (1994) and Hess et al. (1998) is used. Finally, we describe cirrus and clouds by their top and base heights, and cloud optical thickness at $2315 \mathrm{~nm}$. We assume that cirrus fully overcasts the observed scene, whereas broken cloud coverage is addressed by the independent pixel approximation (Marshak et al., 1995). Moreover, we assume the US Standard Atmosphere (NOAA, 1976) for the profiles of dry air density, pressure, water and $\mathrm{CO}$. The $\mathrm{CH}_{4}$ profile is taken from the European background scenario of Levelt et al. (2009).

The radiance spectra are perturbed by measurement noise from the TROPOMI noise model by Tol et al. (2011) for an instantaneous view with a footprint of $3.5 \times 7 \mathrm{~km}^{2}$ and a telescope aperture of $12 \mathrm{~mm}^{2}$. (The etendue of the SWIR channel is $4.3 \times 10^{-10} \mathrm{~m}^{2} \mathrm{sr}$.) The optical transmittance of the instrument is adjusted such that, for a spectral sampling of $0.1 \mathrm{~nm}$, a signal-to-noise ratio of 100 is achieved in the continuum of the spectrum for a dark reference scene (surface albedo $A_{\mathrm{s}}=0.05$, viewing zenith angle $\mathrm{VZA}=0^{\circ}$ and solar zenith angle $\mathrm{SZA}=70^{\circ}$ ). The instrument noise includes noise due to the thermal background, the dark current of the detector, the readout noise and the analog-to-digital converter noise.

Figure 4 shows an example of the $\mathrm{CO}$ retrieval performance for simulated measurements with increasing cloud coverage over land and a dark land surface with an albedo $A_{\mathrm{S}}=0.05$. It depicts the retrieval bias $b_{\mathrm{CO}}$, the retrieval noise $\sigma_{\mathrm{CO}}$, and the column averaging kernel. The retrieval biases increases to $2.3 \%$ with increasing cloud fraction because deficits of our cloud model become more relevant with increasing cloud coverage. At the same time, the retrieval noise of the CO column decreases due to the gain in the measurement signal. The change of the retrieval sensitivity with cloud coverage is clearly illustrated by the column averaging kernels shown in the right panel of Fig. 4. When the cloud frac- tion is greater than zero, the column averaging kernel starts to increase above the cloud and at the same time decreases below the cloud, and so reflects the effect of cloud shielding on the retrieved column utilizing the profile scaling approach (Borsdorff et al., 2014).

Similar results were already presented by Vidot et al. (2012), who used a previous version of the SICOR algorithm. In their study, clouds were accounted for in the retrieval by an elevated Lambertian reflector. This approach appeared to be appropriate to describe the effect of optically thick clouds, and boundary layer aerosols in the retrieval and similar small retrieval biases are achieved with the latest version of SICOR described here. However, in case of an optically thin scattering layer due to an elevated dust layer, optically thin clouds and cirrus above a bright surface, the previous version of SICOR (Vidot et al., 2012) could not account for any path enhancement of the observed light due to light trapping between the scattering layer and the surface. In the study of Vidot et al. (2012), this shortcoming became clear when assessing the retrieval accuracy for optically thin cirrus above bright surfaces. This is the main reason why the twostream radiative transfer solver is used in the current algorithm, which approximates both transmission and reflection of a cloud and so allows for photon trapping between optically thin clouds and a bright surface. In the following, our analysis focuses on these new aspects of our algorithm.

\subsection{Forward model errors}

The forward model of our retrieval introduces errors due to the accuracy of the two-stream model, the neglect of atmospheric Rayleigh scattering and the description of clouds and aerosols by a single triangular scattering layer. To elicit the impact of these approximations, we consider three generic measurement ensembles for a clear sky atmosphere and for a cloudy atmosphere with optically thin clouds and cirrus.

Figure 5 shows the $\mathrm{CO}$ retrieval bias and the corresponding retrieval noise for simulated clear sky measurements including atmospheric Rayleigh scattering with a variable surface albedo and a variable solar zenith angle. Overall, the retrieval bias is small with $-0.5 \% \leq b_{\mathrm{CO}} \leq 0.5 \%$. The retrieval noise increases from values $<1 \%$ at high sun and for bright surfaces to $\approx 11 \%$ for low sun $\left(\mathrm{SZA}=70^{\circ}\right)$ and low albedo $\left(A_{\mathrm{s}}=0.03\right)$. This increase is governed by the signal strength and so by the signal-to-noise ratio of the measurement.

To investigate the effect of photon trapping between clouds and the surface, Fig. 6 depicts the $\mathrm{CO}$ bias for a cloud between 4 and $5 \mathrm{~km}$ altitude with a small optical depth $\tau_{\text {scat }}=2$ as a function of surface albedo and cloud coverage. Here, the $\mathrm{CO}$ bias reaches $1.5 \%$ with increasing cloud coverage. For a cirrus layer between 9 and $10 \mathrm{~km}$ of varying optical depth as function of the surface albedo, the light trapping effect at high surface albedo results in a $\mathrm{CO}$ biases $b_{\mathrm{CO}} \leq 0.5 \%$. 

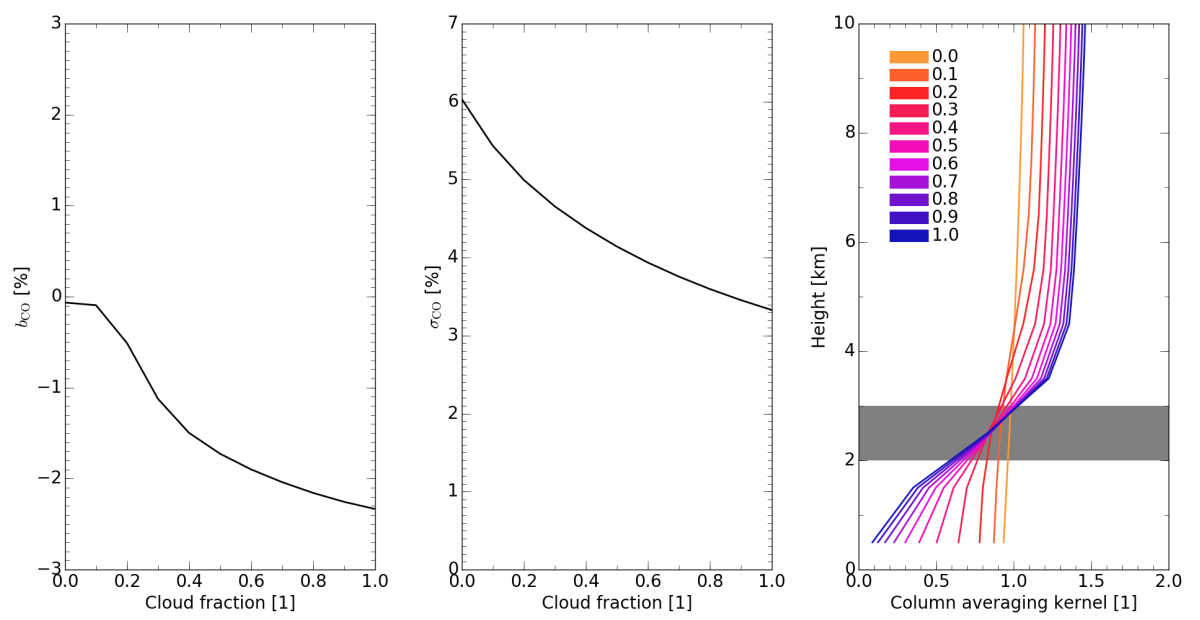

Figure 4. Example of the S5P CO data product and its performance as a function of cloud fraction $f_{\text {cld }}$. The SWIR measurements are simulated for a scene partially covered by a cloud between 2 and $3 \mathrm{~km}$ with optical depth $\tau_{\mathrm{cld}}=5$, a surface albedo $A_{\mathrm{s}}=0.05$, a solar zenith angle of $50^{\circ}$ and a viewing zenith angle of $40^{\circ}$. Left panel: CO retrieval bias $b_{\mathrm{CO}}$. Middle panel: retrieval noise $\sigma_{\mathrm{CO}}$. Right panel: column averaging kernel for different cloud fractions as indicated in the legend. The gray area indicates the position of the cloud.
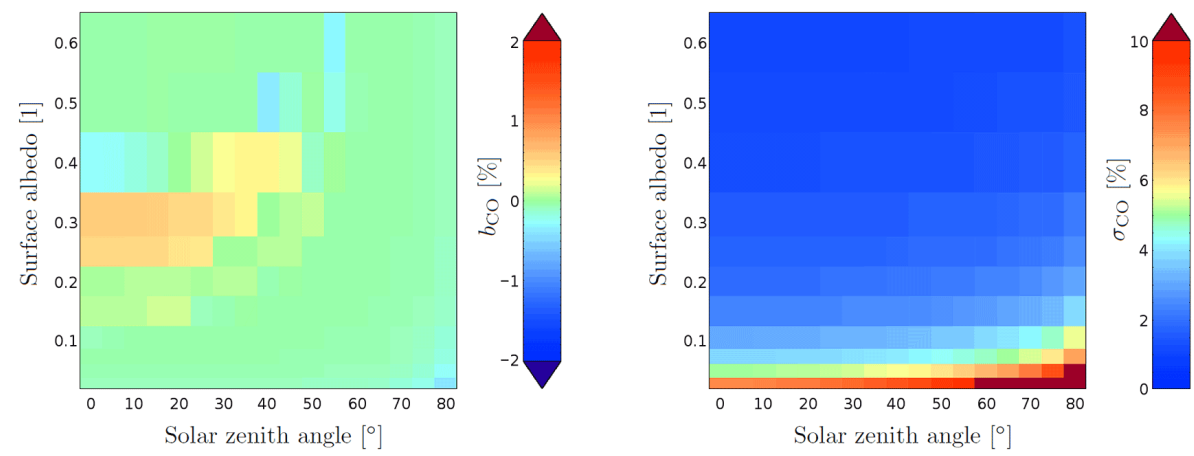

Figure 5. Retrieval bias $b_{\mathrm{CO}}$ (left panel) and retrieval noise $\sigma_{\mathrm{CO}}$ (right panel) for the clear sky conditions (without aerosol, clouds and cirrus) and for a viewing zenith angle (VZA) of $0^{\circ}$ as a function of solar zenith angle (SZA) and surface albedo $A_{\mathrm{S}}$.

Similar small biases are found for an elevated dust layer and optically thin clouds (not shown).

Moreover, we investigated the implications of the retrieved cloud parameters being effective cloud parameters. These parameters differ from the truth because of the limited information available from the satellite measurements. Here, the retrieval forward model has to describe clouds in a simplified manner with a few free parameters, and all remaining cloud properties have to be fixed a priori (see e.g., Koelemeijer et al., 2002; van Diedenhoven et al., 2007). In our case, the cloud model includes several simplifications, e.g., a horizontally homogenous cloud with the triangular height distribution in optical depth, and a two-stream radiative transfer model to describe the cloud radiative properties. Considering the measured radiometric signal as a mean of a photon ensemble with different light paths through the atmosphere, the retrieval adjusts the cloud parameters and the simulated light paths such that the methane absorption features can be fitted by the forward model. This may include erroneous light paths, of which effects average out in the simulated measurement for the particular height distribution of methane. However for another trace gas with a different vertical profile, such as $\mathrm{CO}$, the relevance of the individual photons for the observed signal may differ, and so the simulated light paths introduce spectral errors in the simulated CO absorption features. Subsequently adjusting the trace gas concentrations in the retrieval, $\mathrm{CO}$ biases are introduced for cloudy atmospheres.

Obviously, this retrieval error depends on the particular $\mathrm{CO}$ profile and the altitude at which the simulated light path deviates from its truth. Therefore, to characterize this inherent bias of our retrieval approach, we simulate SWIR measurements for a cloudy atmosphere adding $\mathrm{CO}$ abundance in a $1 \mathrm{~km}$ thick, vertically homogenous layer with varying layer top height $z_{\text {per }}$. Here, the $\mathrm{CO}$ enhancement increases the $\mathrm{CO}$ total column by $50 \%$. Figure 7 shows the $\mathrm{CO}$ biases as a 

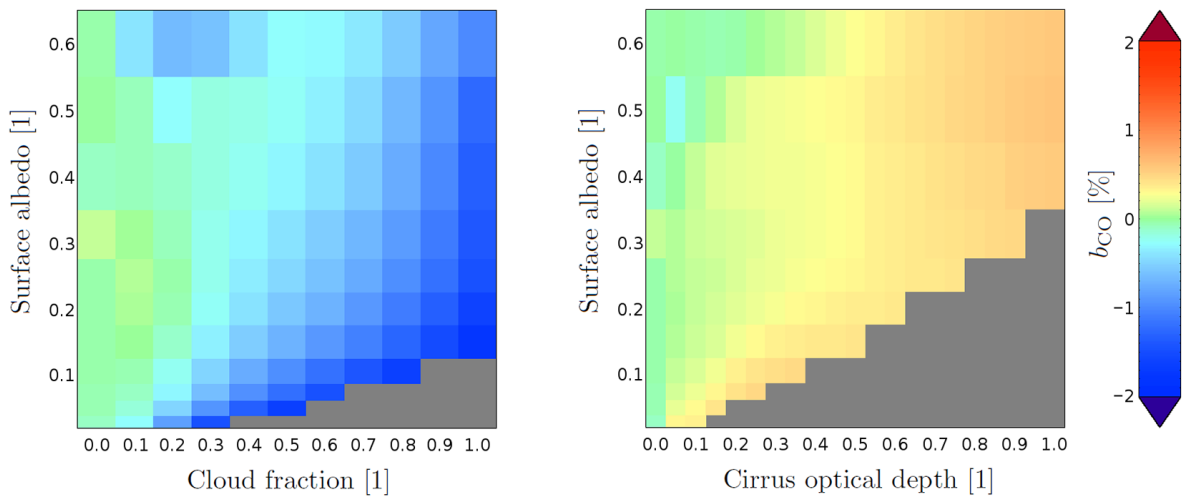

Figure 6. Left panel: retrieval bias in case of a cloud atmosphere. The CO bias is shown as a function of surface albedo $A_{\mathrm{S}}$ and cloud fraction $f$ for a cloud between 4 and $5 \mathrm{~km}$ altitude with optical depth $\tau_{\text {scat }}=2$ and a VZA of $0^{\circ}$. Right panel: CO retrieval bias for measurements in presence of optically thin cirrus, which overcasts the entire scene, as a function of surface albedo and cirrus optical depth that defined at $2300 \mathrm{~nm}$. The gray area indicates measurement simulations, which were rejected by the cloud filter.

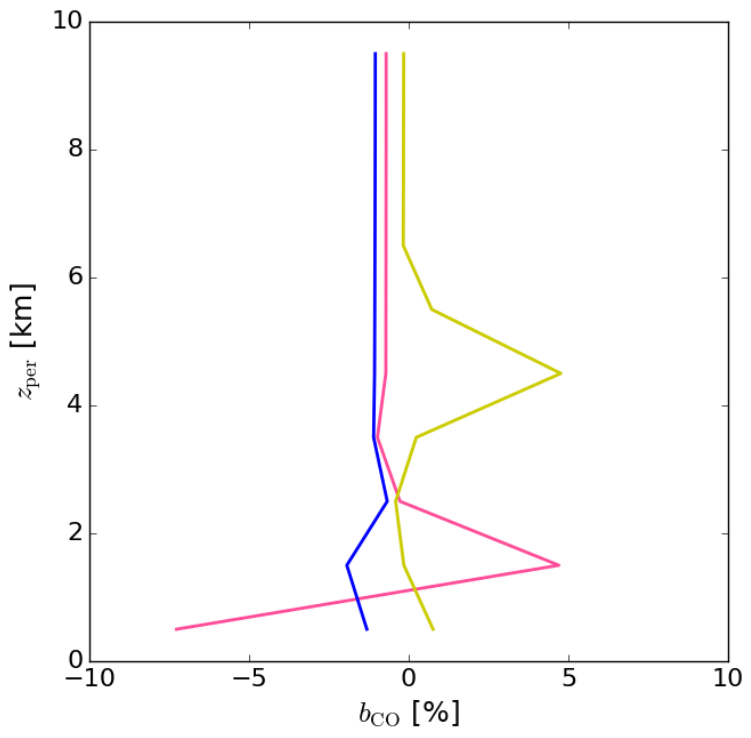

Figure 7. Retrieval bias $b_{\mathrm{CO}}$ in cloudy atmospheres in case of strongly enhanced $\mathrm{CO}$ concentrations. Measurement simulations are performed for a surface albedo $A_{\mathrm{s}}=0.05$, SZA and VZA of 50 and $0^{\circ}$ and for overcast sky with a cloud at $1-2 \mathrm{~km}$ altitude with an optical depth of $\tau_{\text {cld }}=2$ (pink) and $\tau_{\text {cld }}=5$ (blue). Additionally, we consider a case of partially cloud cover with cloud fraction $f_{\text {cld }}=0.1$ at $4-5 \mathrm{~km}$ altitude with $\tau_{\text {cld }}=2$ (yellow). The CO profile represents the US Standard Atmosphere with a perturbation at the indicated altitude $z_{\text {per }}$ enhancing the total amount of $\mathrm{CO}$ by $50 \%$.

function of $z_{\text {per }}$ for scenes covered with low clouds at $1-2 \mathrm{~km}$ altitude with optical thicknesses of 2 and 5, and a cloud at 4$5 \mathrm{~km}$ covering $10 \%$ of the scene with a cloud optical thickness of 2. In each case, the simulated measurement passes the cloud filter of Sect. 2.1. We clearly see a positive retrieval bias up to $5 \%$ for enhanced $\mathrm{CO}$ concentration at the altitude of the optically thin cloud, whereas a negative bias of $7 \%$ is found for low clouds in combination with a near-surface $\mathrm{CO}$ enhancements. The latter error is relevant for burning events localized in the tropospheric boundary layer. Above the cloud, the error sensitivity is only small, indicating that the light path at this altitude range is well described by our simplified radiative transfer model. Furthermore, for the optically thicker clouds the error sensitivity is below $2 \%$, as expected for a primarily reflecting cloud.

\subsection{Atmospheric parameters}

An important element of the CO retrieval approach is the use of methane a priori information to determine effective cloud properties from the SWIR measurements as discussed above. The SICOR retrieval relies on simulated $\mathrm{CH}_{4}$ fields from the TM5 model (Krol et al., 2005), which have been used in several studies (e.g., Meirink et al., 2008; Bergamaschi et al., 2005, 2009). Via the inverse modeling technique, the sources and sinks of $\mathrm{CH}_{4}$ in the TM5 model are optimized by minimizing the residual differences between model and measurements from the NOAA-ESRL global monitoring network and deviations from the a priori surface flux distribution (Houweling et al., 2014). In the following, we refer to these model runs as the TM5-NOAA simulations.

To test the overall accuracy of the model simulations, we compare 1 year of $\mathrm{CH}_{4}$ model fields with collocated GOSAT observations (Butz et al., 2009, 2010, 2011; Schepers et al., 2012). Here, the GOSAT $\mathrm{CH}_{4}$ product is extensively validated with TCCON ground measurements with an overall root-mean-square (rms) difference of $15 \mathrm{ppb}$ and a stationto-station bias of $3.5 \mathrm{ppb}$ (Detmers and Hasekamp, 2015). Within these boundaries, the GOSAT $\mathrm{XCH}_{4}$ retrieval can be used to estimate the model accuracy. To this end, Fig. 8 shows the difference between GOSAT- and TM5-NOAAsimulated $\mathrm{XCH}_{4}$. Over China, the largest biases of up to $3 \%$ occur because of inconsistencies in the underlying emission scenario in combination with a limited regional coverage of 


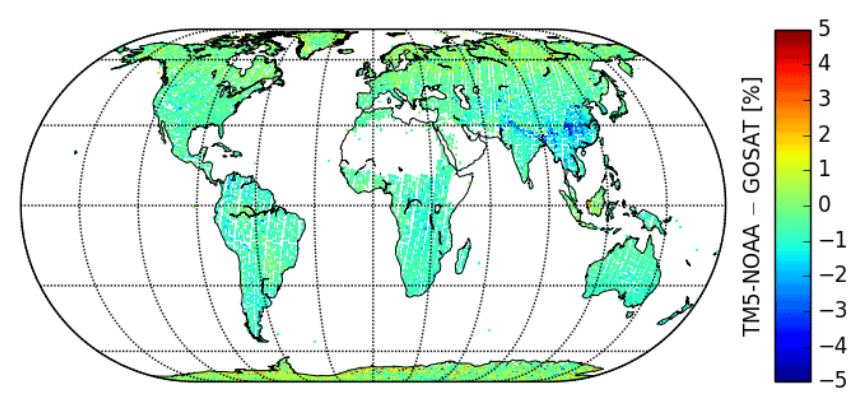

Figure 8. Difference between $\mathrm{CH}_{4}$ total column dry air mixing ratios from TM5-NOAA simulations and GOSAT retrievals for the period June 2009 to December 2012.

the NOAA-ESRL ground-based measurements. Overall biases are smaller with an rms difference between GOSAT and TM5-NOAA, amounting to $20 \mathrm{ppb}$ and increasing towards southern latitudes. This latitudinal bias in TM5, relative to GOSAT, is also found in other models (see e.g., Locatelli et al., 2015) and is currently under further investigation. Comparisons of the modeled $\mathrm{CH}_{4}$ columns with collocated TCCON measurements are largely consistent with these findings with an rms difference between 8 and $22 \mathrm{ppb}$ depending on the TCCON site.

Inherent to this analysis is the assumption that the NOAAESRL measurements are available in a timely manner to perform model simulation as input to the retrieval. This timeliness of the simulation needs further consideration. Commonly, inverse-modeling-derived estimates lag behind real time by approximately 1 year. This is mostly due to the availability of various types of inputs that are required, including meteorological fields, a priori emission estimates, and measurements. Due to that, we propose a modeling procedure that uses the inversion-optimized TM5 estimates of the dry air mole column mixing ratio of methane $\mathrm{XCH}_{4}$ of the previous year. Obviously, the largest error source is the variability in $\mathrm{XCH}_{4}$ caused by the year-to-year variations in meteorology and the interannual variability of the methane sources and sinks. We estimate the size of the error from results of a multi-year inversion for the period 2003-2010, calculating how $\mathrm{XCH}_{4}$ on a given day of the year (15 January, April, July and October) varied between the years. Largest variations are found over Southeast Asia, due to large regional sources of methane, but errors in the meteorology of the northern and southern hemispheric storm tracks are also present. On average, the standard deviations are well within $1 \%$ (18 ppb) on average, regionally increasing up to $1.5 \%$ (27 ppb). Sporadically, standard deviations up to $3 \%$ are found, associated with biomass burning events. Acknowledging these limitations in our approach, an uncertainty of $3 \%$ of our methane a priori knowledge seems a reasonable margin that should be achievable for most conditions encountered throughout the global domain.

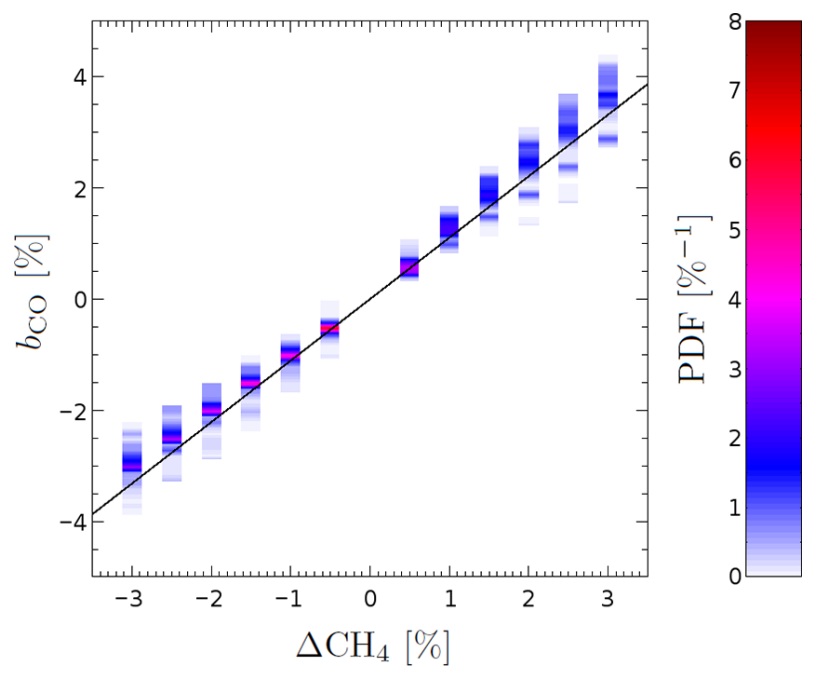

Figure 9. $\mathrm{CO}$ bias due to a priori errors in $\mathrm{CH}_{4}$ for the clear sky measurement ensemble of Fig. 5. For each $\mathrm{CH}_{4}$ error, the $\mathrm{CO}$ bias probability function is shown. The $\mathrm{CO}$ error sensitivity is estimated by a linear regression through all data points (solid line).

For the generic clear sky measurement ensembles, Fig. 9 shows the PDF of the $\mathrm{CO}$ biases as a function of the methane model error of approximately $\pm 3 \%$. A linear regression through the data points indicates a nearly one-to-one error correspondence with $1.11 \% \mathrm{CO}$ bias due to $1 \%$ error in the methane model columns. Table 1 provides the corresponding bias sensitivity for the cloudy and cirrus measurement ensembles in Fig. 6. Aggregating these results, we conclude that the $\mathrm{CO}$ retrieval bias due to the uncertainty of the TM5NOAA model input typically does not exceed $3 \%$.

Additionally to the $\mathrm{CH}_{4}$ a priori error, an erroneous surface pressure affects the inferred $\mathrm{CO}$ column both through a wrong conversion of the methane mixing ratio $\mathrm{XCH}_{4}$ into the total column density of methane and via an erroneous spectroscopy because of the pressure broadening of individual absorption lines. For the operational retrieval, we use pressure information from the European Centre for Medium-Range Weather Forecasts (ECMWF) with a typical accuracy of 2$3 \mathrm{hPa}$ (Salstein et al., 2008). Subsequently, ECMWF surface pressure is interpolated on the particular TROPOMI pixel by means of the digital elevation map of Farr (2007) and Danielson and Gesch (2011), accounting for the topography of the terrain. For pressure uncertainties in the range $\leq 3 \mathrm{hPa}$, we obtain an error sensitivity of $0.11-0.13 \%$ CO column error per $1 \mathrm{hPa}$ surface pressure error for the clear sky and cloudy scenarios of our generic measurement ensemble. Furthermore, we evaluated the impact of uncertainties in the atmospheric temperature forecast of ECMWF, which has been estimated at a few Kelvin. Table 1 lists the CO retrieval sensitivities with respect to an offset of the atmospheric temperature profile in the range $\pm 3 \mathrm{~K}$, which vary between 0.17 and $0.23 \%$ CO column error per $1 \mathrm{~K}$ temperature offset. Thus 
Table 1. CO column retrieval sensitivity in $\%$ with respect to the uncertain knowledge of a set of atmospheric and instrument parameters for the generic clear sky, cloud and cirrus ensemble: (1) $\mathrm{CH}_{4}$ a priori uncertainty of TM5-NOAA runs, (2) ECMWF surface pressure uncertainty, (3) ECMWF temperature profile offset, (5) FWHM uncertainty of the ISRF, (6) spectral calibration error $\delta s$ and (7) the radiometric offset $I_{\mathrm{offset}}$ and a multiplicative radiometric error $I_{\text {scal }}$ of the level 1 data product.

\begin{tabular}{llrrr}
\hline No. & Parameter & Clear sky & Cloud & Cirrus \\
\hline 1 & $\mathrm{CH}_{4}$ a priori $\left(\% \%^{-1}\right)$ & 1.11 & 1.18 & 1.21 \\
2 & pressure $\left(\% \mathrm{hPa}^{-1}\right)$ & 0.11 & 0.13 & 0.13 \\
3 & temperature $\left(\% \mathrm{~K}^{-1}\right)$ & 0.23 & 0.17 & 0.20 \\
4 & FWHM $\left(\% \%^{-1}\right)$ & 0.51 & 0.40 & 0.43 \\
5 & $\delta s\left(\% 10 \mathrm{pm}^{-1}\right)$ & 0.88 & 0.87 & 0.87 \\
6 & $I_{\text {offset }}\left(\% \%^{-1}\right)$ & -0.63 & -0.47 & -0.46 \\
7 & $I_{\text {scal }}\left(\% \%^{-1}\right)$ & 0.01 & 0.01 & 0.02 \\
\hline
\end{tabular}

for the $\mathrm{CO}$ column product, we expect the corresponding retrieval biases due to inaccuracies in the atmospheric parameters to be well within $1 \%$.

\subsection{Instrument effects}

Finally, we studied the $\mathrm{CO}$ retrieval sensitivity with respect to a set of instrument-related parameters. First, the Earth radiance spectrum may be subject to a radiometric offset $I_{\mathrm{offset}}$, expressed relative to the radiance level at the reference wavelength of $2315 \mathrm{~nm}$, or a spectrally constant multiplicative error $\delta I_{\text {scal }}$. Instrumental reasons for these errors can be manyfold, e.g., uncorrected stray light, detector and read-out electronics performance and an erroneous pre-flight instrument calibration. For the generic ensembles, we derived an error sensitivity of -0.47 to $-0.63 \% \mathrm{CO}$ column error per percent radiometric offset and 0.01 to $0.02 \%$ per percent multiplicative radiometric error. The corresponding TROPOMI observation requirement for a radiometric offset is $<0.1 \%$, and for a multiplicative radiometric error on the Earth radiance measurement it is $<2 \%$ (Buscaglione, 2011). The main reason for this robust $\mathrm{CO}$ retrieval performance with respect to this type of radiometric errors is the selected spectral window with relatively weak atmospheric absorption. Here, these spectral biases can be mitigated efficiently by the retrieval of an effective surface albedo and cloud properties.

To study an erroneous spectral calibration of the measurement, we assumed a correct instrument calibration $\lambda_{i}$ of spectral detector $i$ and an erroneous calibration

$\lambda_{i}^{\prime}=\lambda_{i}+\frac{\lambda_{i}-\lambda_{m}}{\lambda_{r}-\lambda_{m}} \delta s$.

Here, $\lambda_{r}=2385 \mathrm{~nm}$ indicates the longwave edge of the SWIR band and $\lambda_{m}=2345 \mathrm{~nm}$ is the spectral center. Therefore, $\delta s$ characterizes the spectral calibration errors at the edges of the SWIR spectral range, whereas in the center $\lambda_{m}$ the calibration error vanishes. The corresponding spectral squeeze for the CO fit windows $(2315-2338 \mathrm{~nm})$ is about one-third of $\delta s$. The error sensitivity of the CO column product is about $0.9 \%$ per $\delta s=10 \mathrm{pm}$. Due to the required knowledge of the center of all SWIR channels of $<2 \mathrm{pm}$ (Langen et al., 2011), this CO error sensitivity is not critical for a compliant instrument. Moreover, the $\mathrm{CO}$ retrieval has no error sensitivity to an overall offset of the spectral calibration because this parameter is adjusted by the retrieval.

Errors in the instrument spectral response function can be manyfold and are hard to quantify in a general manner. In this study, we restricted ourself to an erroneous full width at half maximum (FWHM) of the instrument spectral response function (ISRF), which may occur e.g., because of pre-flight instrument calibration errors or because of fluctuations of the instrument temperature. Table 1 shows the ISRF retrieval sensitivity of about $0.5 \% \mathrm{CO}$ error for a $1 \%$ FWHM uncertainty of the ISRF, the latter representing the knowledge requirement for the TROPOMI instrument calibration (Langen et al., 2011).

Finally, we discuss the $\mathrm{CO}$ column error contribution originating from radiometric artifacts due to the heterogeneous illumination of the instrument entrance slit, which in turn arises from varying cloud coverage and surface reflection within a spatial sample. As discussed by Noel et al. (2012) and Caron et al. (2014) and in Appendix B, this results in a distortion of the spectral response of the TROPOMI instrument. Accounting for this effect in the retrieval requires, next to detailed characterization of the instrument, a priori knowledge of the radiance heterogeneity across the instrument slit, which is not available. For future instrument development, e.g., for the succeeding Sentinel 5 mission of ESA, this instrumental effect is foreseen to be mitigated by a slit homogenizer (Caron et al., 2014). This is an optical device scrambling the spatial information of the incoming signal in the flight direction, and so the spectrometer is effectively exposed to a spatially homogeneous entrance signal. Because the TROPOMI instrument is not equipped with such a device, it is important to quantify potential errors on the $\mathrm{CO}$ data product.

For this purpose, we considered two spatial ensembles of simulated measurements. First, we investigated a MODIS Aqua cloud image over Australia, shown in Fig. 10, characterizing clouds by a cloud mask on a $1 \times 1 \mathrm{~km}^{2}$ spatial grid box. For each of the samples at a spatial position $(x, y)$, we calculated a spectral radiance field $I(x, y, \lambda)$ assuming a ground albedo $A_{\mathrm{s}}=0.1$ and, depending on the cloud mask, a vertically homogenous cloud between 2 and $3 \mathrm{~km}$ with an optical depth of $\tau_{\text {scat }}=20$. Next, we simulated the TROPOMI observations with the instrument model in Appendix B. Subsequent retrievals allow us to quantify the $\mathrm{CO}$ bias due to the distortion of the instrument response for the ensemble. Results of this test (right panel of Fig. 10) show with a characteristic $\mathrm{CO}$ bias pattern at cloud edges ranging from up to $+2 \%$, where the cloud edge enters the instrument field of 
view, to minimum $-2 \%$ when the field of view points mainly at clouds and the scene heterogeneity is due to a remaining contribution of clear sky radiances. Although this error contribution is significant, it has quasi-random characteristics when looking at larger spatial or temporal domains because of the quasi-random occurrence of clouds on these scales. Additionally, we investigated a measurement ensemble for spatially varying surface albedo of a $50 \times 50 \mathrm{~km}^{2}$ wetland region in Siberia. The albedo distribution is adapted from MODIS Aqua observation at $2.1 \mu \mathrm{m}$ with a spatial sampling of $500 \times 500 \mathrm{~m}^{2}$, with a mean albedo of 0.037 and a standard deviation of 0.017 (see Fig. 11). The patchy structure of the figure is due to dark ponds of the marsh. Figure 11 also shows a CO bias of about $\pm 1.5 \%$ related to the scene heterogeneity. The mean error of the ensemble reduces to $0.05 \%$ with a standard deviation of $0.44 \%$, supporting the quasi-random characteristics of this error.

\section{Sentinel 5 Precursor orbit ensemble}

To test the algorithm performance and the data product accuracy for more common circumstances encountered in the operational processing, we have simulated a measurement ensemble for a typical TROPOMI orbit, employing the noise model as described in Sect. 3. The simulations are based on a dedicated Sentinel 5 Precursor orbit simulations for August with 13:30 Equator crossing time providing pixel location and size as well as the solar and viewing geometry of the TROPOMI observation (M. Sneep, Royal Netherlands Meteorological Institute, the Netherlands, personal communication, 2016). Here, we only considered pixels with SZA $<80^{\circ}$, for which the TROPOMI instrument performance is constrained by the mission specifications. In the first instance, we spatially projected the trail ensemble by Butz et al. (2010) for the same month to the test orbit to collocated CO and $\mathrm{CH}_{4}$ concentrations from TM5 (S. Houweling, SRON, personal communication, 2016) and $\mathrm{H}_{2} \mathrm{O}$ from the ECMWF forecasts to the individual TROPOMI pixels. Additionally, we use the aerosol properties from the ECHAM5-HAM model (Stier and et al., 2005) and monthly mean MODIS observations (Remer, 2005). The cirrus optical thickness is specified to match the CALIOP monthly median cirrus optical thickness and height distribution (Winker et al., 2007). The surface albedo is taken from the global SCIAMACHY albedo database at $2350 \mathrm{~nm}$ (Butz et al., 2012). Finally, we overlaid the ensemble with the MODIS Aqua cloud product comprising cloud top height, cloud fraction and cloud optical depth for the individual spatial samplings of the orbit. Hence, the measurement ensemble includes a variety of TROPOMI viewing and solar geometries, combined with realistic variations of atmospheric scattering and trace gas abundances. Figure 12 shows examples of the atmospheric parameters in the ensemble.
Table 2. Fraction of data to be processed during the successive processor steps for the TROPOMI test orbit ensemble relative to the 572442 spectra of the orbit ensemble that are filtered for $\mathrm{SZA}<80^{\circ}$.

\begin{tabular}{lll}
\hline Processor step & Process & $R(\%)$ \\
\hline 1 & SZA < $80^{\circ}$ & 100 \\
2 & LER filtering & 87 \\
2 & $\mathrm{CH}_{4}$ cloud filtering & 46 \\
4 & check convergence & 38 \\
5 & retrieval noise filter & 36 \\
\hline
\end{tabular}

The operational processing sequence starts with rejecting all observations with a signal that is too low based on the Lambert-equivalent reflectivity, defined as follows:

$\mathrm{LER}=\max _{i}\left\{\frac{I^{\mathrm{TOA}}\left(\lambda_{i}\right) \pi}{\mu_{0} F_{0}\left(\lambda_{i}\right)}\right\}$,

where $F_{0}\left(\lambda_{i}\right)$ is the solar irradiance at spectral samplings $\lambda_{i}$. The maximum is taken over all spectral samplings within the CO fitting window. For measurements with LER $>0.03$, we assess the cloud filter described in Sect. 2.1. Figure 12 shows the clear correlation of $\Delta \mathrm{CH}_{4}$ in panel d with the cloud parameters in panels a, b and c. For our test orbit, about $46 \%$ of the data passed the cloud filter $\left|\Delta \mathrm{CH}_{4}\right|<25 \%$. This is significantly less than for the 1 year of GOSAT observations in Fig. 3, indicating a particularly cloudy test orbit. In the next processing step, we retrieved the CO total column together with the effective cloud properties as described in Sect. 2.1. The final data quality of our $\mathrm{CO}$ product is further enhanced by an a posteriori quality filter accepting only retrievals with a retrieval noise $\sigma_{\mathrm{CO}}<12 \%$. It is important to realize that the chosen filter thresholds give a first indication of the data processing statistics, based on the expected instrument performance. However, during the commissioning phase of TROPOMI, further adjustments will be required. For our test orbit, about $36 \%$ of all data successfully passed the processing. Table 2 summarizes the relative number of data that pass the individual steps. The corresponding $\mathrm{CO}$ retrieval bias is depicted in panel $\mathrm{f}$ of Fig. 12, which indicates an overall good quality of our algorithm. However, a clear feature is present in central Africa with a negative bias of about $-8 \%$. It coincides with enhanced $\mathrm{CO}$ concentrations from biomass burning regions as shown in panel e of the same figure. For these observations, the $\mathrm{CO}$ concentration in the atmospheric boundary layer is strongly enhanced, and so the $\mathrm{CO}$ profiles differs significantly in shape from that of $\mathrm{CH}_{4}$. As discussed in the previous section, for these circumstances we expect a systematic underestimation of $\mathrm{CO}$ for low-cloud conditions, which is confirmed by the orbit simulations.

For the observations that pass all quality filters, we analyzed the orbit simulations in more detail looking at the PDF of the $\mathrm{CO}$ bias together with $\Delta \mathrm{CH}_{4}$. The density function 

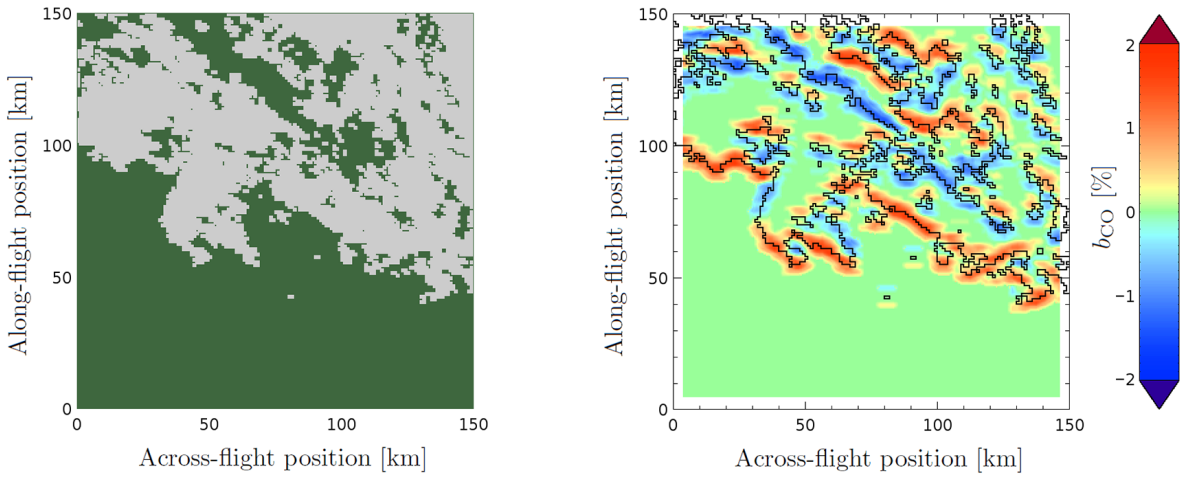

Figure 10. Error due to heterogeneous slit illumination due to a cloudy scene over Australia, 12 February 2010. Left panel: cloud mask derived from MODIS Aqua observations at $1 \times 1 \mathrm{~km}^{2}$ spatial sampling (green indicates clear sky pixels; gray indicates cloud flagged pixels). Right panel: CO retrieval bias due to heterogeneous illumination of the instrument entrance slit. The cloudy areas are indicated by the black contour line.
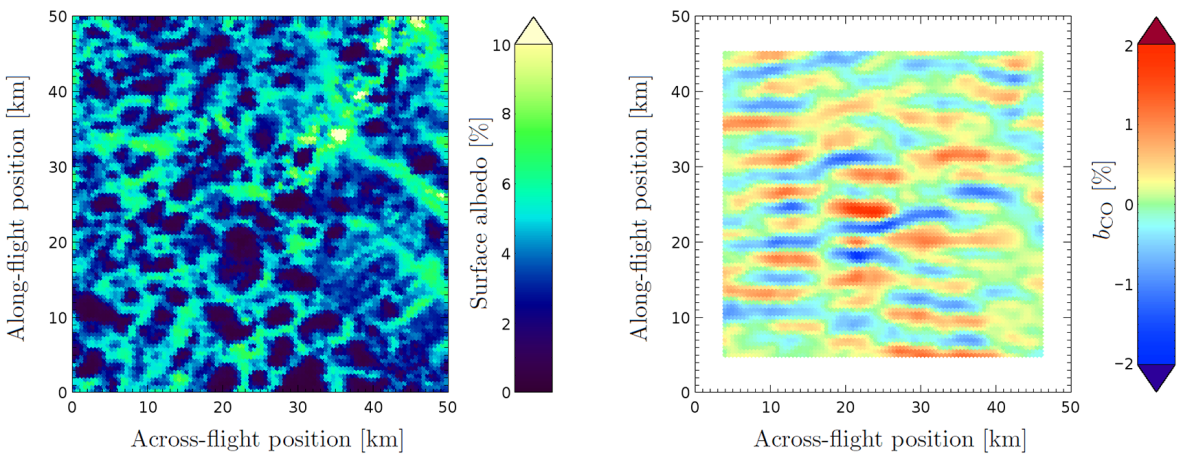

Figure 11. Error due to heterogeneous slit illumination by a scene with varying surface reflection over a marsh scene in Siberia close to the river $\mathrm{Ob}$ at latitude $62.8^{\circ} \mathrm{N}$ and longitude $72.1^{\circ} \mathrm{E}$. Left panel: MODIS Lambertian albedo at $2.1 \mu \mathrm{m}$ with a spatial sampling of $0.5 \times 0.5 \mathrm{~km}{ }^{2}$. Right panel: $\mathrm{CO}$ retrieval error due to heterogeneous illumination of the instrument entrance slit.

of $\Delta \mathrm{CH}_{4}$ is depicted in Fig. 13 and has a maximum around zero representing clear sky scenes. The tail towards negative $\Delta \mathrm{CH}_{4}$ values comprises cloudy observations, and positive values indicate cases of light path enhancements due to atmospheric scattering. The corresponding distribution of the $\mathrm{CO}$ bias shows a weak dependence on $\Delta \mathrm{CH}_{4}$ and therefore on cloud coverage. This nicely demonstrates the functional capability of our retrieval algorithm for a suite of different atmospheric conditions. Overall for the orbit ensemble, the mean $\mathrm{CO}$ bias is $0.9 \%$ with a standard deviation of $1.1 \%$, which is well within the envisaged retrieval accuracy.

Finally, we roughly estimated the computational performance of the algorithm for a HP dc7900 SFF workstation with Intel ${ }^{\circledR}$ Core $^{\text {TM }} 2$ Duo 1390 CPU E8400 at $3.00 \mathrm{GHz}$, with a floating point rate of 237 and 4 GB RAM. Numerical experiments showed the computational burden of a single $\mathrm{CO}$ retrieval to be $0.17 \mathrm{~s}$ using the Intel FORTRAN compiler. Thus, to keep up with the TROPOMI data acquisition rate, parallel processing is required on at least 22 processor cores.

\section{Summary and conclusions}

In this paper, we presented the baseline algorithm for the operational CO data processing of the Sentinel 5 Precursor mission. The algorithm relies on a two-step retrieval from TROPOMI SWIR measurements. First, we perform a non-scattering retrieval of the total amount of $\mathrm{CH}_{4}$ in the spectral range $2315-2324 \mathrm{~nm}$ for cloud filtering. In the presence of high and optically thick clouds, the inferred $\mathrm{CH}_{4}$ column differs significantly from its true value, which is used together with modeled methane abundances to filter TROPOMI observations accordingly. Further processing only considers measurements with differences of the non-scattering methane column and the model prediction of $\left|\Delta \mathrm{CH}_{4}\right|<25 \%$. The CO column is inferred from SWIR measurements in the adjacent spectral window 2324 $2338 \mathrm{~nm}$. In this step, we use a priori knowledge on the atmospheric methane abundance to retrieve effective cloud parameters simultaneously with atmospheric $\mathrm{CO}$ and $\mathrm{H}_{2} \mathrm{O}$ abundances. The algorithm employs a profile scaling approach to infer the $\mathrm{CO}$ total column amount and a two-stream radiative 
(a)

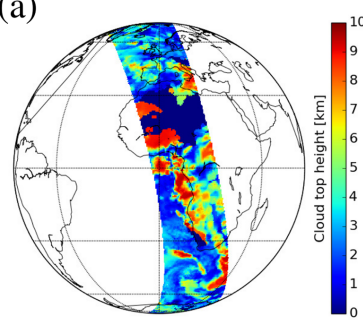

(c)

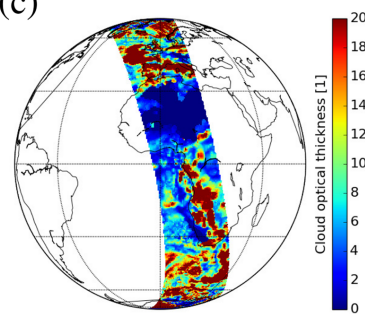

(e)

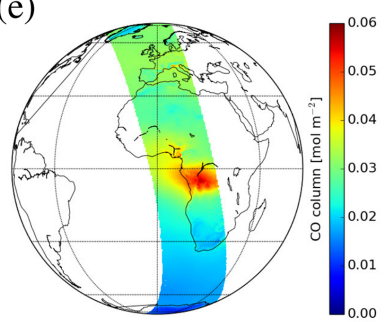

(b)

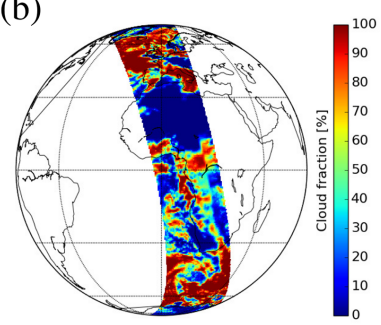

(d)

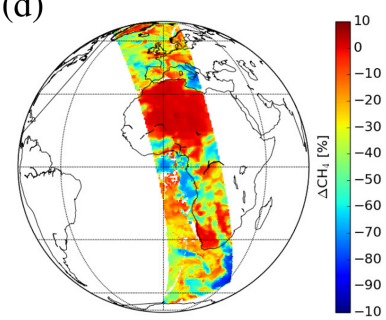

(f)

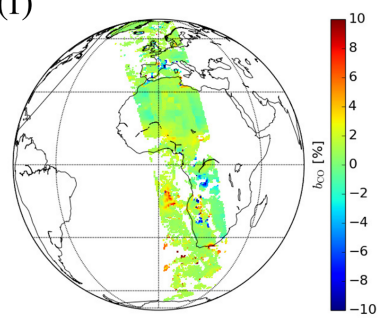

Figure 12. TROPOMI test orbit. Panel (a) cloud top height, panel (b) cloud fraction, panel (c) cloud optical depth, panel (d) methane error of non-scattering retrieval, which is used for cloud filtering, panel (e) TM4 CO total column, panel (f) $\mathrm{CO}$ retrieval bias.

transfer model that is linearized with respect to the parameters to be retrieved. The two-stream approach is a simple approximation to account for multiple light scattering, and its numerical implementation has a low computational cost. The vertically integrated $\mathrm{CO}$ column density is provided together with its retrieval noise and the column averaging kernel for each individual measurement. This compact retrieval product is designed to address the needs of the data user, while taking optimal advantage of the SWIR measurements.

To demonstrate the robustness of our algorithm and the expected data quality of the $\mathrm{CO}$ retrieval product, we performed an extensive sensitivity analysis for generic measurement simulations with respect to forward model errors, instrument and calibration imperfections and uncertainties in atmospheric input parameters. For this purpose, we have simulated measurements with the scalar LINTRAN radiative transfer model, which accurately accounts for multiple scattering of solar light by liquid water and ice clouds, aerosols and the interaction with a reflecting Earth surface. The measurement simulations are fed through the TROPOMI instrument model to estimate the measurement noise. For clear sky

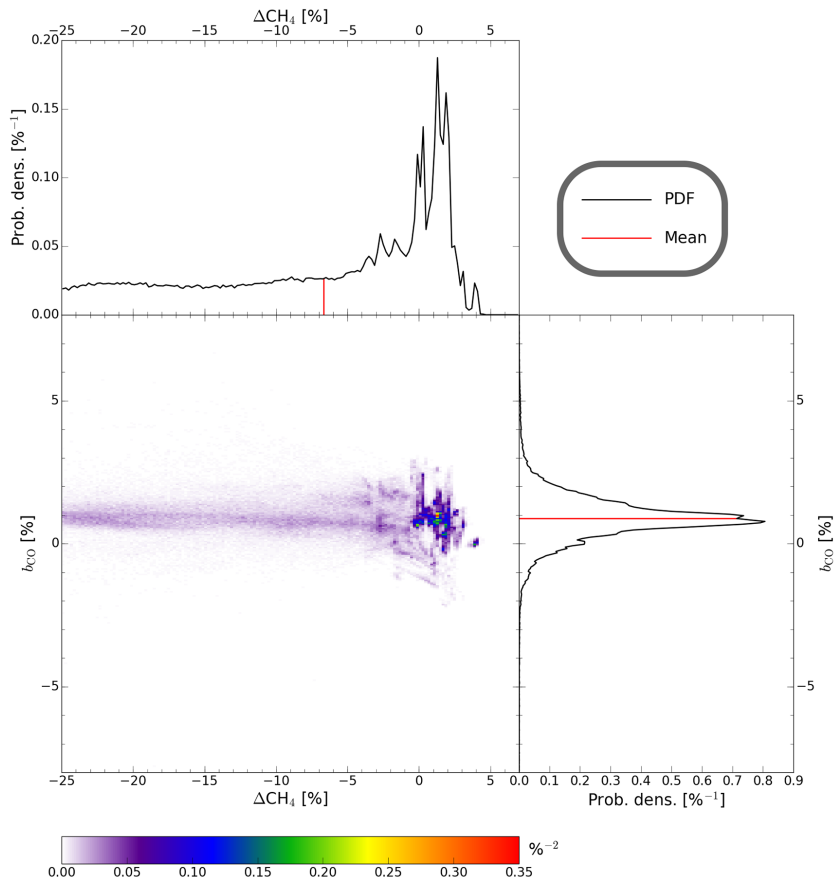

Figure 13. Middle panel: two-dimensional probability density function of the methane filter $\left(\Delta \mathrm{CH}_{4}\right)$ and the $\mathrm{CO}$ retrieval bias $\left(b_{\mathrm{CO}}\right)$. Upper panel: one-dimensional probability density function of $\Delta \mathrm{CH}_{4}$ (mean: $-6.7 \%$, standard deviation: $8.4 \%$ ). Right panel: one-dimensional probability density function of $b_{\mathrm{CO}}$ (mean: $0.9 \%$, standard deviation: $1.1 \%$ ).

scenes of low signals over dark land with $3 \%$ surface albedo and no aerosol loading, the random error in total column $\mathrm{CO}$ does not exceed $11 \%$ for $\mathrm{SZA}<70^{\circ}$, and in the majority of all cases the $\mathrm{CO}$ data precision is expected to be much better. Moreover, for measurement simulations employing the US standard model atmosphere with a single cloud layer, which passed the cloud filter, we diagnosed the retrieval accuracy to be $<2 \%$. Similar good accuracy could be achieved for boundary layer aerosols and elevated dust layers. However, for cloudy atmospheres and strongly peaked $\mathrm{CO}$ vertical profiles, e.g., enhanced $\mathrm{CO}$ concentration in the tropospheric boundary layer, this bias can reach $8 \%$. Concerning the atmospheric input parameters, the largest uncertainties are introduced by model uncertainties in the methane fields. Here, we found a nearly one-to-one correlation between the $\mathrm{CO}$ column error and the $\mathrm{CH}_{4}$ a priori uncertainty, introducing $\mathrm{CO}$ biases $\leq 3 \%$. Uncertainties in the atmospheric temperature and pressure are of minor relevance. To estimate the effect of an erroneous instrument calibration, we considered errors in the full width at half maximum of the ISRF for homogenous illumination of the instrument entrance slit, erroneous spectral calibration and additive and multiplicative radiometric errors. For the TROPOMI instrument that satisfies the mission requirements, corresponding $\mathrm{CO}$ biases are $<1 \%$. A heterogeneous illumination of the instrument en- 
trance slit due to variations in cloud coverage and surface reflection causes a distortion of the spectral instrument response, which we cannot account for in the retrieval. This causes $\mathrm{CO}$ biases $< \pm 2 \%$ with pseudo-random characteristics on larger spatial scales. Overall, the low error sensitivity of the $\mathrm{CO}$ product is also confirmed by a retrieval analysis for a simulated orbit of TROPOMI SWIR measurements. For this purpose, we combined a suite of different data sources to describe the observed scene in a realistic manner. Here, the $\mathrm{CO}$ biases are in agreement with the generic test cases and confirm that the expected retrieval accuracy is well within the envisaged accuracy of $<15 \%$.

Although our analysis is based on an extensive set of simulated measurements, we realize the need to further fine-tune the settings of our algorithm during the commissioning phase of the TROPOMI instrument, aiming to provide an optimal data product during the operational phase of the Sentinel 5 Precursor mission. For this purpose, the validation of the data product with independent and accurate ground-based, balloon and aircraft measurements is essential until instrument commissioning and beyond during the operational phase of the mission to adequately assess and monitor data quality.

\section{Data availability}

The underlying research data for the simulated atmospheric profiles and TROPOMI measurement simulation and retrievals are available upon request from Jochen Landgraf (j.landgraf@sron.nl). HITRAN spectroscopic line parameters (Rothman et al., 2009) are available through HITRANonline (http://hitran.org), and the line parameters from Scheepmaker et al. (2013) are available in their supplementary material. 
Appendix A: TS-LINTRAN: a linearized two-stream method

This appendix summarizes the linearized two-stream radiative transfer solver TS-LINTRAN that is based on the generalized flux method (Meador and Weaver, 1998) and the forward-adjoint perturbation theory (Marchuk, 1964; Bell and Glasstone, 1970; Box et al., 1988; Ustinov, 1991). The solver is part of the software suite LINTRAN, which combines different linearized radiative transfer models suited for atmospheric remote sensing (e.g., Landgraf et al., 2001; Hasekamp and Landgraf, 2002; Landgraf et al., 2002; Walter et al., 2004, 2006; Schepers et al., 2015). The model assumes a vertically inhomogeneous atmosphere described by $N$ homogeneous layers. Each layer is characterized by its optical properties, the optical depth $\tau_{n}$, the single-scattering albedo $\omega_{n}$ and the phase function $P_{n}$ with layer index $n=1, \ldots, N$.

For an arbitrary layer $n$, the outgoing fluxes at the layer interfaces $n-1$ and $n$ can be expressed as a function of the incoming fluxes by the matrix equation (Meador and Weaver, 1998)

$\left(\begin{array}{l}S_{n} \\ F_{n}^{\downarrow} \\ F_{n-1}^{\uparrow}\end{array}\right)=\left(\begin{array}{ccc}a_{1, n} & 0 & 0 \\ a_{2, n} & a_{4, n} & a_{5, n} \\ a_{3, n} & a_{5, n} & a_{4, n}\end{array}\right)\left(\begin{array}{l}S_{n-1} \\ F_{n-1}^{\downarrow} \\ F_{n}^{\uparrow}\end{array}\right)$.

Here, index $n-1=0$ describes the top of the model atmosphere and index $n=N$ indicates the surface level. $S_{n}$ is the direct solar irradiance, $F_{n}^{\downarrow}$ and $F_{n}^{\uparrow}$ are the diffuse downward and upward fluxes, all defined at layer interface $n$. The coefficients $a_{1, n}, a_{2, n}, a_{3, n}, a_{4, n}$ and $a_{5, n}$ are specific for different flux methods, where TS-LINTRAN relies on the definition of the practical improved flux method by Zdunkowski et al. (1979). The external boundary conditions are given as

$S_{0}=\mu_{0} F_{0}$

$F_{0}^{\downarrow}=0$

$F_{N}^{\uparrow}=A_{\mathrm{s}}\left(F_{N}^{\downarrow}+S_{N}\right)$,

where $A_{\mathrm{s}}$ is the surface albedo and $\mu_{0}=\cos \left(\Theta_{0}\right)$ with the solar zenith angle $\Theta_{0}$. Combining the internal and external boundary constraints for the multi-layer system, we obtain the matrix equation

$\mathbf{M} \boldsymbol{F}=\boldsymbol{C}$,

with the sparse block-diagonal matrix $\mathbf{M}$, the flux vector

$\boldsymbol{F}=\left(\begin{array}{cccccc}S_{0}, & F_{0}^{\downarrow}, & F_{0}^{\uparrow}, & S_{1}, \cdots, & S_{N}, & F_{N}^{\downarrow}, F_{N}^{\uparrow}\end{array}\right)^{T}$

and the right-hand side

$\boldsymbol{C}=\left(\begin{array}{llll}\mu_{0} F_{0}, & 0, & \cdots & 0\end{array}\right)^{T}$.

For an $N$-layer model atmosphere, $\mathbf{M}$ is a $3(N+1) \times 3(N+1)$ matrix and $\boldsymbol{F}$ and $\boldsymbol{C}$ are both vectors of dimension $3(N+1)$.
Due to the block diagonal structure of matrix M, Eq. (A3) can be solved by sequential substitution of the linear equations.

With the flux vector $\boldsymbol{F}$, we can approximate the TOA radiances $I^{\mathrm{TOA}}$ in the viewing direction of the instrument. For this purpose we start with the expression

$$
\begin{aligned}
I^{\mathrm{TOA}=} & \frac{F_{N}^{\uparrow}}{\pi} \exp \left(-\tau_{\text {tot }} / \mu_{v}\right)+ \\
& \frac{1}{\mu_{v}} \int_{0}^{\tau_{\text {tot }}} \mathrm{d} \tau J\left(\tau, \mu_{v}\right) \exp \left(-\tau / \mu_{v}\right),
\end{aligned}
$$

where $\mu_{v}=\cos \left(\Theta_{v}\right)$ with the viewing zenith angle $\Theta_{v}, \tau$ indicates optical depth and $\tau_{\text {tot }}$ is the total optical thickness of the atmosphere. The scattering source function $J$ describes multiply and singly scattered light. We approximate the radiance within a model layers by its vertical mean and assume its directional dependence to be isotropic both in upward and downward directions. Therefore, we obtain

$F_{\downarrow \uparrow}=\frac{F_{n-1}^{\downarrow \uparrow}+F_{n}^{\downarrow \uparrow}}{2}$

for $\tau_{n-1}<\tau<\tau_{n}$. Hence, we can approximate Eq. (A6) by

$I^{\mathrm{TOA}}=\langle\boldsymbol{R} \mid \boldsymbol{F}\rangle$,

where the response vector $\boldsymbol{R}$ can de derived in a straightforward manner from Eq. (A6). It describes the linear relationship between the simulated observation and the internal radiation field. Here, the inner product of two arbitrary vectors $\boldsymbol{u}$ and $\boldsymbol{v}$ of the same dimension is defined by $\langle\boldsymbol{u} \mid \boldsymbol{v}\rangle=\boldsymbol{u}^{T} \boldsymbol{v}$.

To apply the forward-adjoint perturbation theory, we solve the adjoint equation

$\mathbf{M}^{T} \boldsymbol{F}^{\dagger}=\boldsymbol{R}$,

where $\boldsymbol{F}^{\dagger}$ is the adjoint flux vector, and $\mathbf{M}^{T}$ is the transpose of matrix M. Following the methodology described by Ustinov (1991) and Walter et al. (2004), we can calculate the derivative of the TOA radiance with respect to an optical parameter $x$ by

$\frac{I^{\mathrm{TOA}}}{\partial x}=\left\langle\boldsymbol{F}^{\dagger} \mid \mathbf{M}^{\prime} \boldsymbol{F}\right\rangle+\left\langle\boldsymbol{F}^{\dagger} \mid \boldsymbol{C}^{\prime}\right\rangle+\left\langle\boldsymbol{R}^{\prime} \mid \boldsymbol{F}\right\rangle$,

(A10)

with the derivatives $\mathbf{M}^{\prime}=\frac{\partial}{\partial x} \mathbf{M}, \boldsymbol{C}^{\prime}=\frac{\partial}{\partial x} \boldsymbol{C}$ and $\boldsymbol{R}^{\prime}=\frac{\partial}{\partial x} \boldsymbol{R}$. With $\boldsymbol{C}$ given in Eq. (A5), the derivative $\boldsymbol{C}^{\prime}$ vanishes, and so Eq. (A10) simplifies to

$\frac{\partial I^{\mathrm{TOA}}}{\partial x}=\left\langle\boldsymbol{F}^{\dagger} \mid \mathbf{M}^{\prime} \boldsymbol{F}\right\rangle+\left\langle\boldsymbol{R}^{\prime} \mid \boldsymbol{F}\right\rangle$.

In general, $x$ represents the optical depth $\Delta \tau_{n}$, the singlescattering albedo $\omega_{n}$, the scattering phase function characteristics in the model layers $n=1, \cdots, N$ and the surface albedo $A_{\mathrm{s}}$. Equation (A11) can be numerically implemented in a straightforward manner, and represents the basis of the linearized TS-LINTRAN solver. 


\section{Appendix B: The heterogeneous slit illumination: an instrument model}

TROPOMI is a push-broom grating spectrometer that measures the spatial and spectral distribution of the Earthreflected radiances using a two-dimensional detector device. Here, the width of the entrance slit is aligned with the flight direction, and after dispersion by the grating, a two-dimensional detector simultaneously collects the spectra from the $2600 \mathrm{~km}$ instrument swath, sampled by 256 rows of the detector. The spectral information is recorded by the 1024 pixel detector columns with a spectral sampling distance of $0.1 \mathrm{~nm}$. The instantaneous field of view of the spectrometer is $3.4 \times 7 \mathrm{~km}^{2}$ (along $\times$ across flight direction), and after temporal integration over $1 \mathrm{~s}$, TROPOMI samples the ground scene with about $7 \times 7 \mathrm{~km}^{2}$ at the subsatellite point. Figure B1 gives an overview of the measurement principle. For the TROPOMI data analysis, we assume that the spectral and spatial dimension of the radiance field can be fully disentangled. However, this is only true for ground scenes that reflect spatially homogenous radiances in the flight direction. If the radiances vary on spatial subsampling scales, we obtain interferences of the scene heterogeneity with the spectral response of the instrument. This appendix summarizes an instrument model that describes the effect of the heterogeneous slit illumination on the recorded spectrum using preliminary TROPOMI instrument characteristics.

The radiometric calibrated signal $S$ measured by TROPOMI can be simulated by

$$
\begin{aligned}
S(\rho, \sigma, \tau)= & \iiint \mathrm{d} x \mathrm{~d} y \mathrm{~d} \lambda U(\rho \mid x, \lambda) V(\sigma \mid y) \frac{1}{t_{\text {int }}} \\
& \int_{t_{l}}^{t_{r}} \mathrm{~d} t I(x-v t, y, \lambda),
\end{aligned}
$$

where $\rho$ and $\sigma$ describe the spectral and spatial sampling position on the two-dimensional detector plane, respectively, and $\tau$ is the temporal sampling. The ground coordinates are $x$ in the along-track direction and $y$ in the across-track direction, and $\lambda$ denotes the wavelength of the light. Due to the orientation of the instrument entrance slit, the $x$ and $y$ directions are identical to the across- and along-slit direction at the instrument level, respectively. In Eq. (B1), $U$ and $V$ denote the instrument response of the recorded signal in the along- and across-flight direction with respect to the radiation $I$ at position $(x, y)$ and at wavelength $\lambda$. Here, our notation separates sampling variables and physical coordinates by a vertical bar. The temporal integration of the received signal

$\langle I\rangle_{t}(\tau \mid x, y, \lambda)=\frac{1}{t_{\text {int }}} \int_{t_{l}}^{t_{r}} \mathrm{~d} t I(x-v t, y, \lambda)$

between $t_{l}=\tau-\frac{1}{2} t_{\text {int }}$ and $t_{r}=\tau+\frac{1}{2} t_{\text {int }}$ corresponds to a spatial integration of the radiances due to the motion of the satel-

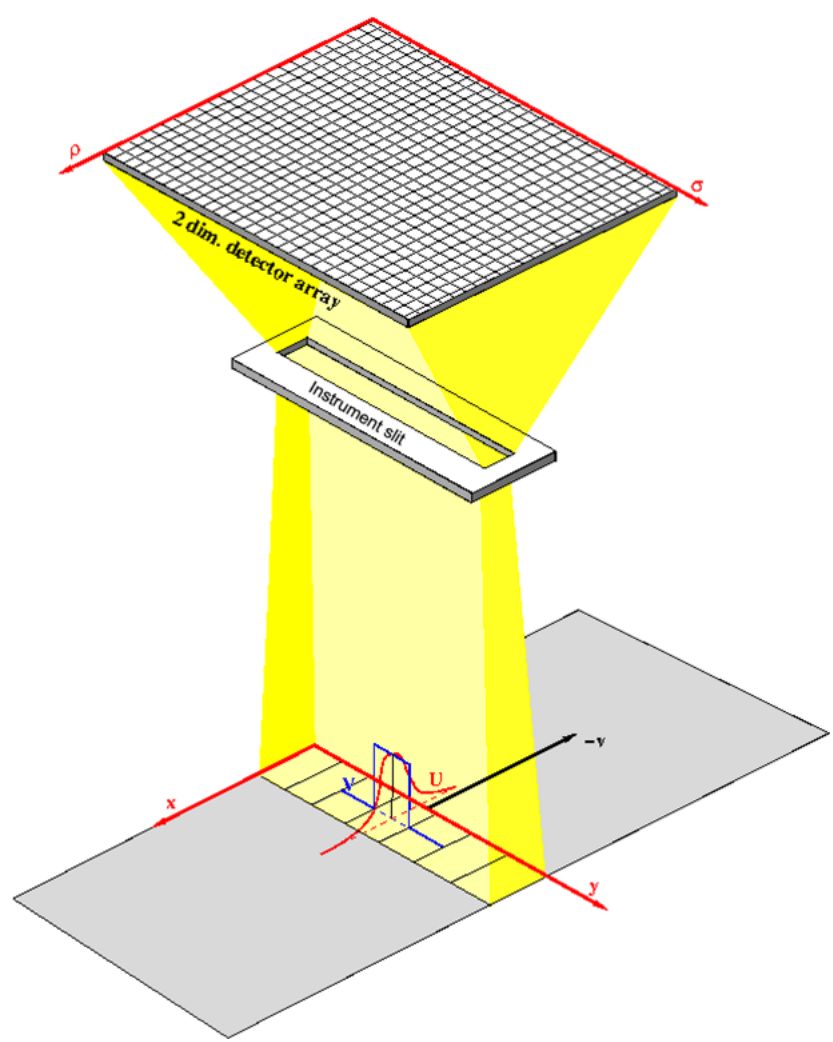

Figure B1. Slit and detector geometry with respect to the ground track of TROPOMI. Detector coordinate $\rho$ describes the spectral sampling dimension, and $\sigma$ is the spatial sampling coordinate. The scene coordinates are $x$ in the flight direction and $y$ in the acrossflight direction, and $U$ and $V$ represent the corresponding instrument response in both spatial dimensions. The slit is aligned with the TROPOMI swath, such that scene heterogeneity in the flight direction interferes with the spectral response of the instrument.

lite, where $t_{\text {int }}$ is the total integration time and $v$ is the satellite velocity on ground level.

For a homogenous illumination of the instrument across the slit direction, i.e., $\langle I\rangle_{t}(\tau \mid x, y, \lambda)=\langle I\rangle_{t}(\tau \mid y, \lambda)$, Eq. (B1) simplifies to

$S(\rho, \sigma, \tau)=\int \mathrm{d} \lambda\langle U\rangle_{x}(\rho \mid \lambda)\langle I\rangle_{y, t}(\sigma, \tau \mid \lambda)$.

Here, the mean intensity

$\langle I\rangle_{y, t}(\sigma, \tau \mid \lambda)=\int \mathrm{d} y V(\sigma \mid y)\langle I\rangle_{t}(\tau \mid x, y, \lambda)$

includes the temporal integration and the convolution of the radiances with the instrument response $V$ across the flight direction. Moreover, we defined the integrated instrument spectral response function in the flight direction

$\langle U\rangle_{x}(\rho \mid \lambda)=\int \mathrm{d} x U(\rho \mid x, \lambda)$,

which is extensively characterized during the on-ground calibration of the TROPOMI spectrometer. Equation (B3) is the 

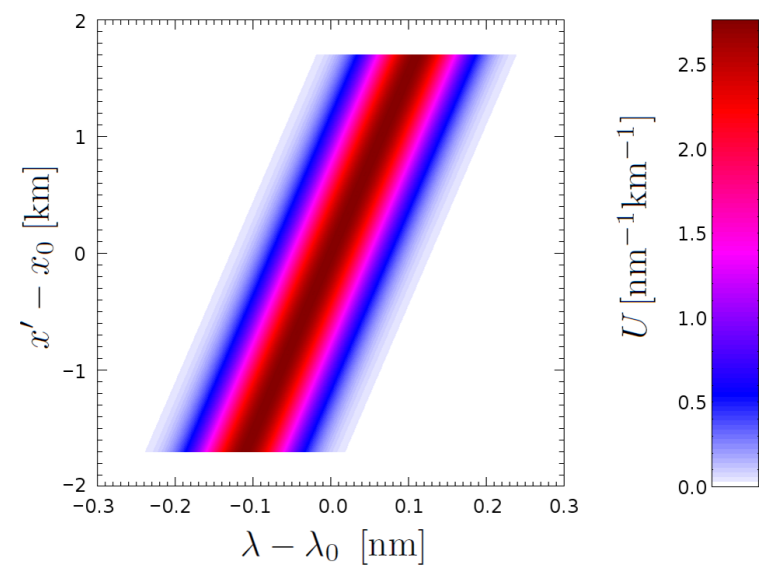

Figure B2. Instrument response function $U\left(x_{0}, \lambda_{0} \mid x, \lambda\right)$ as defined in Eqs. (B7) and (B8) with $\Delta x=3.4 \mathrm{~km}, \Delta w=0.1 \mathrm{~nm}$ and $b=$ $6.47 \times 10^{-2} \mathrm{~nm} \mathrm{~km}^{-1}$.

baseline for our forward model in the retrieval, which assumes an inherent homogenous illumination of the entrance slit. Thus, the differences between the measurement simulations using Eqs. (B1) and (B3) represent a potential error source for the $\mathrm{CO}$ retrieval.

To simplify the further elaboration of the response functions, we assign the sampling variable to spatial and spectral coordinates: we appoint the spatial sampling variable $\sigma$ to the barycenter $y_{0}$ of the instantaneous field of view $V$. Similarly, the spectral sampling $\rho$ is assigned to the barycenter $\lambda_{0}$ of the integrated spectral response function $\langle U\rangle_{x}$, and finally, the barycenter $x_{0}$ of $U$ for $\lambda=\lambda_{0}$ is also assigned a sampling position $\rho$. Obviously, the variables $x_{0}$ and $\lambda_{0}$ are not independent.

Based on the design of the instrument and a preliminary analysis of the on-ground calibration, we assume that the response function $U$ can be factorized, i.e.,

$U\left(x_{0}, \lambda_{0} \mid x, \lambda\right)=U_{1}\left(x_{0} \mid x\right) U_{2}\left(x_{0}, \lambda_{0} \mid x, \lambda\right)$,

where

$U_{1}\left(x_{0} \mid x\right)=\frac{1}{\Delta x} \Theta\left(x-x_{0}-\frac{\Delta x}{2}\right) \Theta\left(x_{0}+\frac{\Delta x}{2}-x\right)$

describes the geometric projection of the slit width on the Earth surface with $\Delta x=3.4 \mathrm{~km}$ and

$$
\begin{aligned}
& U_{2}\left(x_{0}, \lambda_{0} \mid x, \lambda\right)= \\
& \frac{1}{\sqrt{2 \pi} \sigma} \exp \left(-\frac{1}{2 \sigma^{2}}\left(\lambda_{0}-\lambda-b\left(x_{0}-x\right)\right)^{2}\right)
\end{aligned}
$$

is the Gaussian subsampling spectral response function with $\sigma=\frac{\Delta w}{2 \sqrt{2 \ln 2}}$, and the FWHM $\Delta w=0.1 \mathrm{~nm}$. Parameter $b=$ $6.47 \times 10^{-2} \mathrm{~nm} \mathrm{~km}^{-1}$ gives the shift of the spectral barycenter with across-slit position $\left(x_{0}-x\right)$. Therefore, for the homogenous slit illumination, the instrument spectral response

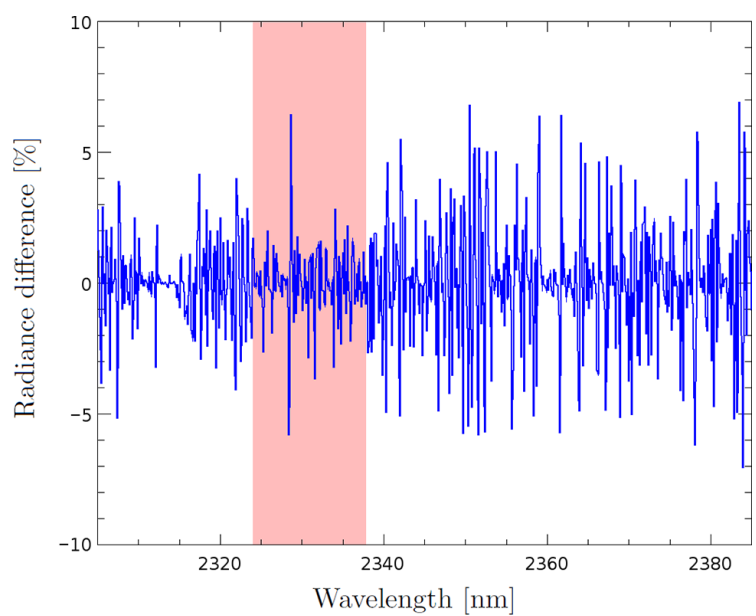

Figure B3. Spectral features due to the inhomogeneous slit illumination as a percentage of the continuum value. Simulations are performed for a transition in the flight direction from a cloudy scene to a clear sky scene at $+1.8 \mathrm{~km}$ away from barycenter $x_{0}$. Here, the cloud is located between 2 and $3 \mathrm{~km}$ with a total optical depth of 10 . The CO fitting window is indicated by the pink shadowed region.

function $\langle U\rangle_{x}$ is a convolution of a Gaussian fit with a boxcar function and has a FWHM of $0.25 \mathrm{~nm}$, according to the instrument requirement. The response function $U$ is illustrated in Fig. B2. For the spatial response function across-flight direction $V$, we assume a boxcar function of $7 \mathrm{~km}$ wide.

To analyze the error of our retrieval, we use Eq. (B3) in the forward model of the retrieval, but simulate the measurements using Eq. (B1), which introduces a spectral bias as depicted exemplarily in Fig. B3. Here, spectral biases are about $\pm 7 \%$, with an error amplitude strongly depending on the assumed scene heterogeneity. 
Acknowledgements. We thank Maarten Sneep (The Royal Netherlands Meteorological Institute, KNMI) for providing us with auxiliary data needed to simulate TROPOMI measurements. We also thank Bernd Sierk and Jerome Caron (European Space Research and Technology Centre, ESTEC) for constructive discussions on modeling the effect of the heterogenous illumination of the instrument entrance slit. This research has been funded in part by the TROPOMI national program from the Netherlands Space Office (NSO).

Edited by: R. Engelen

Reviewed by: two anonymous referees

\section{References}

Aben, I., Hasekamp, O., and Hartmann, W.: Uncertainties in the space-based measurements of $\mathrm{CO}_{2}$ columns due to scattering in the Earth's atmosphere, J. Quant. Spectrosc. Ra., 104, 450-459, doi:10.1016/j.jqsrt.2006.09.013, 2007.

Bell, G. I. and Glasstone, S.: Nuclear Reactor Theory, Van Nostrand Reinhold Company, New York, ISBN-10: 0442206844, ISBN13: $9780442206840,1970$.

Bergamaschi, P., Krol, M., Dentener, F., Vermeulen, A., Meinhardt, F., Graul, R., Ramonet, M., Peters, W., and Dlugokencky, E. J.: Inverse modelling of national and European $\mathrm{CH}_{4}$ emissions using the atmospheric zoom model TM5, Atmos. Chem. Phys., 5, 2431-2460, doi:10.5194/acp-5-2431-2005, 2005.

Bergamaschi, P., Frankenberg, C., Meirink, J.-F., Krol, M., Gabriella Villani, M., Houweling, S., Dentener, F., Dlugokencky, E. J., Miller, J. B., Gatti, L. V., Engel, A., and Levin, I.: Inverse modeling of global and regional $\mathrm{CH}_{4}$ emissions using SCIAMACHY satellite retrievals, J. Geophys. Res., 114, D22301, doi:10.1029/2009JD012287, 2009.

Borsdorff, T., Hasekamp, O. P., Wassmann, A., and Landgraf, J.: Insights into Tikhonov regularization: application to trace gas column retrieval and the efficient calculation of total column averaging kernels, Atmos. Meas. Tech., 7, 523-535, doi:10.5194/amt7-523-2014, 2014.

Borsdorff, T., Tol, P., Williams, J. E., de Laat, J., aan de Brugh, J., Nédélec, P., Aben, I., and Landgraf, J.: Carbon monoxide total columns from SCIAMACHY $2.3 \mu \mathrm{m}$ atmospheric reflectance measurements: towards a full-mission data product (2003-2012), Atmos. Meas. Tech., 9, 227-248, doi:10.5194/amt-9-227-2016, 2016.

Bovensmann, H., Burrows, J. P., Buchwitz, M., Frerick, J., Noel, S., Rozanov, V. V., Chance, K. V., and Goede, A. P. H.: SCIAMACHY: Mission Objectives and Measurement Modes, J. Atmos. Sci., 56, 127-150, 1999.

Box, M., Gerstl, S., and Simmer, C.: Application of the adjoint formulation to the calculation of atmospheric radiative effects, Beitr. Phys. Atmos., 61, 303-311, 1988.

Buchwitz, M., de Beek, R., Noël, S., Burrows, J. P., Bovensmann, H., Schneising, O., Khlystova, I., Bruns, M., Bremer, H., Bergamaschi, P., Körner, S., and Heimann, M.: Atmospheric carbon gases retrieved from SCIAMACHY by WFM-DOAS: version $0.5 \mathrm{CO}$ and $\mathrm{CH}_{4}$ and impact of calibration improvements on $\mathrm{CO}_{2}$ retrieval, Atmos. Chem. Phys., 6, 2727-2751, doi:10.5194/acp6-2727-2006, 2006.
Buchwitz, M., Khlystova, I., Bovensmann, H., and Burrows, J. P.: Three years of global carbon monoxide from SCIAMACHY: comparison with MOPITT and first results related to the detection of enhanced CO over cities, Atmos. Chem. Phys., 7, 2399 2411, doi:10.5194/acp-7-2399-2007, 2007.

Buscaglione, F.: GMES Sentinel-5 Precursor - S5p System Requirement Document, Srd, ESA, S5p-RS-ESA-SY-0002, 29 April 2011

Butz, A., Hasekamp, O., Frankenberg, C., and Aben, I.: Retrievals of atmospheric $\mathrm{CO}_{2}$ from simulated space-borne measurements of backscattered near-infrared sunlight: accounting for aerosol effects, Appl. Opt., 48, 3322, doi:10.1364/AO.48.003322, 2009.

Butz, A., Hasekamp, O., Frankenberg, C., Vidot, J., and Aben, I.: $\mathrm{CH}_{4}$ retrievals from spacebased solar backscatter measurements: performance evaluation against simulated aerosol and cirrus loaded scenes, J. Geophys. Res., 115, D24302, doi:10.1029/2010JD014514, 2010.

Butz, A., Guerlet, S., Hasekamp, O., Schepers, D., Galli, A., Aben, I., Frankenberg, C., Hartmann, J. M., Tran, H., Kuze, A., Keppel-Aleks, G., Toon, G., Wunch, D., Wennberg, P., Deutscher, N., Griffith, D., Macatangay, R., Messerschmidt, J., Notholt, J., and Warneke, T.: Toward accurate $\mathrm{CO}_{2}$ and $\mathrm{CH}_{4}$ observations from GOSAT, Geophys. Res. Lett., 38, L14812, doi:10.1029/2011GL047888, 2011.

Butz, A., Galli, A., Hasekamp, O., Landgraf, J., Tol, P., and Aben, I.: TROPOMI aboard Sentinel-5 Precursor: Prospective performance of $\mathrm{CH}_{4}$ retrievals for aerosol and cirrus loaded atmospheres, Remote Sens. Environ., 120, 267-276, 2012.

Caron, J., Sierk, B., Bezy, J., Loescher, A., and Meijer, Y.: The CarnoSat candidate mission: radiometric and specteral performances over spatially heterogeneouse scenes, in: International Conference on Space Optics, ICOS, Tenerife, Spain, 7-10 October 2014.

Danielson, J. and Gesch, D.: Global multi-resolution terrain elevation data 2010 (GMTED2010), US Geological Survey Open-File Report, 2011-1073, 2011.

Deeter, M., Edwards, D. P., Gille, J. C., and Drummond, J. R.: CO retrievals based on MOPITT near-infrared observations, J. Geophys. Res., 114, D04303, doi:10.1029/2008JD010872, 2009.

Detmers, R. and Hasekamp, O.: Comprehensive Error Characterisation Report: SRON full-physics retrieval algorithm for $\mathrm{XCH}_{4}$, Report, ESA Climate Change Initiative (CCI), ESA ESRIN, 2015.

Drummond, J. and Mand, G.: The measurements of pollution in the troposphere (MOPITT) instrument: Overall performance and calibration requirements, J. Atmos. Ocean. Tech., 13, 314-320, 1996.

Edwards, D. P., Emmons, L. K., Hauglustaine, D. A., Chu, D. A., Gille, J. C., Kaufman, Y. J., PéTron, G., Yurganov, L. N., Giglio, L., Deeter, M. N., Yudin, V., Ziskin, D. C., Warner, J., Lamarque, J.-F., Francis, G. L., Ho, S. P., Mao, D., Chen, J., Grechko, E. I., and Drummond, J. R.: Observations of carbon monoxide and aerosols from the Terra satellite: Northern Hemisphere variability, J. Geophys. Res., 109, D24202, doi:10.1029/2004JD004727, 2004.

Farr, T. G., Rosen, P. A., Caro, E., Crippen, R., Duren, R., Hensley, S., Kobrick, M., Paller, M., Rodriguez, E., Roth, L., Seal, D., Shaffer, S., Shimada, J., Umland, J., Werner, M., Oskin, M., Burbank, D., and Alsdorf, D.: The Shuttle Radar Topography Mis- 
sion, Rev. Geophys., 45, RG2004, doi:10.1029/2005RG000183, 2007.

Frankenberg, C., Platt, U., and Wagner, T.: Retrieval of CO from SCIAMACHY onboard ENVISAT: detection of strongly polluted areas and seasonal patterns in global $\mathrm{CO}$ abundances, Atmos. Chem. Phys., 5, 1639-1644, doi:10.5194/acp-5-1639-2005, 2005.

Gimeno García, S., Schreier, F., Lichtenberg, G., and Slijkhuis, S.: Near infrared nadir retrieval of vertical column densities: methodology and application to SCIAMACHY, Atmos. Meas. Tech., 4, 2633-2657, doi:10.5194/amt-4-2633-2011, 2011.

Gloudemans, A. M. S., Schrijver, H., Hasekamp, O. P., and Aben, I.: Error analysis for $\mathrm{CO}$ and $\mathrm{CH}_{4}$ total column retrievals from SCIAMACHY $2.3 \mu \mathrm{m}$ spectra, Atmos. Chem. Phys., 8, 39994017, doi:10.5194/acp-8-3999-2008, 2008.

Gloudemans, A. M. S., de Laat, A. T. J., Schrijver, H., Aben, I., Meirink, J. F., and van der Werf, G. R.: SCIAMACHY CO over land and oceans: 2003-2007 interannual variability, Atmos. Chem. Phys., 9, 3799-3813, doi:10.5194/acp-9-3799-2009, 2009.

Hasekamp, O. and Landgraf, J.: A linearized vector radiative transfer model for atmospheric trace gas retrieval, J. Quant. Spectrosc. Ra., 75, 221-238, 2002.

Hess, M. and Wiegner, M.: COP: a data library of optical properties of hexagonal ice crystals, Appl. Opt., 33, 7740-7746, doi:10.1364/AO.33.007740, 1994.

Hess, M., Koelemeijer, R. B. A., and Stammes, P.: Scattering matrices of imperfect hexagonal ice crystals, J. Quant. Spectrosc. Ra., 60, 301-308, doi:10.1016/S0022-4073(98)00007-7, 1998.

Houweling, S., Krol, M., Bergamaschi, P., Frankenberg, C., Dlugokencky, E. J., Morino, I., Notholt, J., Sherlock, V., Wunch, D., Beck, V., Gerbig, C., Chen, H., Kort, E. A., Röckmann, T., and Aben, I.: A multi-year methane inversion using SCIAMACHY, accounting for systematic errors using TCCON measurements, Atmos. Chem. Phys., 14, 3991-4012, doi:10.5194/acp-14-39912014, 2014.

Koelemeijer, R. B. A., Stammes, P., Hovenier, J. W., and de Haan, J. F.: Global distributions of effective cloud fraction and cloud top pressure derived from oxygen A band spectra measured by the Global Ozone Monitoring Experiment: Comparison to ISCCP data, J. Geophys. Res., 107, 4151, doi:10.1029/2001JD000840, 2002.

Krol, M., Houweling, S., Bregman, B., van den Broek, M., Segers, A., van Velthoven, P., Peters, W., Dentener, F., and Bergamaschi, P.: The two-way nested global chemistry-transport zoom model TM5: algorithm and applications, Atmos. Chem. Phys., 5, 417432, doi:10.5194/acp-5-417-2005, 2005.

Kuze, A., Suto, H., Nakajima, M., and Hamazaki, T.: Thermal and near infrared sensor for carbon observation Fouriertrans form spectrometer on the Greenhouse Gases Observing Satellite for greenhouse gases monitoring, Appl. Opt., 48, 6716, doi:10.1364/AO.48.006716, 2009.

Landgraf, J., Hasekamp, O., Box, M., and Trautmann, T.: A Linearized Radiative Transfer Model Using the Analytical Perturbation Approach, J. Geophys. Res., 106, 27291-27305, 2001.

Landgraf, J., Hasekamp, O., and Trautmann, T.: Linearization of radiative transfer with respect to surface properties, J. Quant. Spectrosc. Ra., 72, 327-339, 2002.
Langen, J., Meijer, Y., Brinksma, E., Veihelmann, B., and Ingmann, P.: GMES Sentinels 4 and 5 mission requirements document, Mrd, ESA, EOP-SMA/1507/JL-dr, 31 January 2011.

Levelt, P., Veefkind, J., Kerridge, B., Siddans, R., de Leeuw, G., Remedios, J., and Coheur, P.: Observation Techniques and Mission Concepts for Atmospheric Chemistry (CAMELOT), Report European Space Agency, Noordwijk, the Netherlands, 2009.

Levy, H.: Normal atmosphere: Large radical and formaldehyde concentrations predicted, Science, 173, 141-143, 1971.

Locatelli, R., Bousquet, P., Saunois, M., Chevallier, F., and Cressot, C.: Sensitivity of the recent methane budget to LMDz subgrid-scale physical parameterizations, Atmos. Chem. Phys., 15, 9765-9780, doi:10.5194/acp-15-9765-2015, 2015.

Logan, J., Prather, M., Wofsy, S., and McElroy, M.: Tropospheric chemistry: A global perspective, J. Geophys. Res., 86, 7210 7254, 1981.

Marchuk, G.: Equation for the Value of Information from Weather Satellites and Formulation of Inverse Problems, Cosmic Res., 2, 394-409, 1964.

Marshak, A., Davis, A., Wiscombe, W., and Titov, G.: The Verisimilitude of the Independent Pixel Approximation Used in Cloud Remote Sensing, Remote Sens. Environ., 52, 71-78, 1995.

Meador, W. E. and Weaver, W. R.: Two-Stream Approximations to Radiative Transfer in Planetary Atmospheres: A Unified Description of Existing Methods and a New Improvement, J. Atmos. Sci., 37, 630-643, 1998.

Meirink, J. F., Bergamaschi, P., and Krol, M. C.: Fourdimensional variational data assimilation for inverse modelling of atmospheric methane emissions: method and comparison with synthesis inversion, Atmos. Chem. Phys., 8, 6341-6353, doi:10.5194/acp-8-6341-2008, 2008.

NOAA: U.S. Standard Atmosphere, 1976, Report, National Oceanic and Atmospheric Administration, Washington, DC, US Gov. Printing Office, 1976.

Noël, S., Bramstedt, K., Bovensmann, H., Gerilowski, K., Burrows, J. P., Standfuss, C., Dufour, E., and Veihelmann, B.: Quantification and mitigation of the impact of scene inhomogeneity on Sentinel-4 UVN UV-VIS retrievals, Atmos. Meas. Tech., 5, 1319-1331, doi:10.5194/amt-5-1319-2012, 2012.

Phillips, P.: A technique for the numerical solution of certain integral equations of the first kind, J. Ass. Comput. Mat., 9, 84-97, 1962.

Predoi-Cross, A., Brawley-Tremblay, M., Brown, L. R., Devi, V. M., and Benner, D. C.: Multispectrum analysis of ${ }^{12} \mathrm{CH}$ 4 from 4100 to $4635 \mathrm{~cm}^{-1}$ : II. Air-broadening coefficients (widths and shifts), J. Mol. Spectrosc., 236, 201-215, doi:10.1016/j.jms.2006.01.013, 2006.

Remer, L. A., Kaufman, Y. J., Tanré, D., Mattoo, S., Chu, D. A., Martins, J. V., Li, R.-R., Ichoku, C., Levy, R. C., Kleidman, R. G., Eck, T. F., Vermote, E., and Holben, B. N.: The MODIS aerosol algorithm, products, and validation, J. Atmos. Sci., 62, 947-973, 2005. Rothman, L. S., Gordon, I. E., Barbe, A., Benner, D. C., Bernath, P. F., Birk, M., Boudon, V., Brown, L. R., Campargue, A., Champion, J.-P., Chance, K., Coudert, L. H., Dana, V., Devi, V. M., Fally, S., Flaud, J.-M., Gamache, R. R., Goldman, A., Jacquemart, D., Kleiner, I., Lacome, N., Lafferty, W. J., Mandin, J.-Y., Massie, S. T., Mikhailenko, S. N., Miller, C. E., Moazzen-Ahmadi, N., Naumenko, O. V., Nikitin, A. V., Orphal, J., Perevalov, V. I., Perrin, A., Predoi-Cross, A., Rinsland, C. P., 
Rotger, M., Šimečková, M., Smith, M. A. H., Sung, K., Tashkun, S. A., Tennyson, J., Toth, R. A., Vandaele, A. C., and Vander Auwera, J.

Rothman, L. S., Gordon, I. E., Barbe, A., Benner, D. C., Bernath, P. F., Birk, M., Boudon, V., Brown, L. R., Campargue, A., Champion, J.-P., Chance, K., Coudert, L. H., Dana, V., Devi, V. M., Fally, S., Flaud, J.-M., Gamache, R. R., Goldman, A., Jacquemart, D., Kleiner, I., Lacome, N., Lafferty, W. J., Mandin, J.-Y., Massie, S. T., Mikhailenko, S. N., Miller, C. E., Moazzen-Ahmadi, N., Naumenko, O. V., Nikitin, A. V., Orphal, J., Perevalov, V. I., Perrin, A., Predoi-Cross, A., Rinsland, C. P., Rotger, M., Šimečková, M., Smith, M. A. H., Sung, K., Tashkun, S. A., Tennyson, J., Toth, R. A., Vandaele, A. C., and Vander Auwera, J.: The HITRAN 2008 molecular spectroscopic database, J. Quant. Spectrosc. Ra., 110, 533-572, doi:10.1016/j.jqsrt.2009.02.013, 2009 (data available at: http: //hitran.org).

Salstein, D. A., Ponte, R. M., and Cady-Pereira, K.: Uncertainties in atmospheric surface pressure fields from global analyses, J. Geophys. Res., 113, D14107, doi:10.1029/2007JD009531, 2008.

Scheepmaker, R. A., Frankenberg, C., Galli, A., Butz, A., Schrijver, H., Deutscher, N. M., Wunch, D., Warneke, T., Fally, S., and Aben, I.: Improved water vapour spectroscopy in the $4174-4300 \mathrm{~cm}^{-1}$ region and its impact on SCIAMACHY $\mathrm{HDO} / \mathrm{H}_{2} \mathrm{O}$ measurements, Atmos. Meas. Tech., 6, 879-894, doi:10.5194/amt-6-879-2013, 2013.

Scheepmaker, R. A., aan de Brugh, J., Hu, H., Borsdorff, T., Frankenberg, C., Risi, C., Hasekamp, O., Aben, I., and Landgraf, J.: $\mathrm{HDO}$ and $\mathrm{H}_{2} \mathrm{O}$ total column retrievals from TROPOMI shortwave infrared measurements, Atmos. Meas. Tech., 9, 39213937, doi:10.5194/amt-9-3921-2016, 2016.

Schepers, D., Guerlet, S., Butz, A., Landgraf, J., Frankenberg, C., Hasekamp, O., Blavier, J.-F., Deutscher, N. M., Griffith, D. W. T., Hase, F., Kyro, E., Morino, I., Sherlock, V., Sussmann, R., and Aben, I.: Methane retrievals from Greenhouse Gases Observing Satellite (GOSAT) shortwave infrared measurements: Performance comparison of proxy and physics retrieval algorithms, J. Geophys. Res., 117, D10307, doi:10.1029/2012JD017549, 2012.

Schepers, D., aan de Brugh, J., Hahne, P., Butz, A., Hasekamp, O., and Landgraf, J.: LINTRAN v2.0: A linearised vector radiative transfer model for efficient simulation of satellite-born nadir-viewing reflection measurements of cloudy atmospheres, J. Quant. Spectrosc. Ra., 149, 247-259, 2015.

Shindell, D. T., Faluvegi, G., Stevenson, D. S., Krol, M. C., Emmons, L. K., Lamarque, J.-F., PéTron, G., Dentener, F. J., Ellingsen, K., Schultz, M. G., Wild, O., Amann, M., Atherton, C. S., Bergmann, D. J., Bey, I., Butler, T., Cofala, J., Collins, W. J., Derwent, R. G., Doherty, R. M., Drevet, J., Eskes, H. J., Fiore, A. M., Gauss, M., Hauglustaine, D. A., Horowitz, L. W., Isaksen, I. S. A., Lawrence, M. G., Montanaro, V., Müller, J.-F., Pitari, G., Prather, M. J., Pyle, J. A., Rast, S., Rodriguez, J. M., Sanderson, M. G., Savage, N. H., Strahan, S. E., Sudo, K., Szopa, S., Unger, N., van Noije, T. P. C., and Zeng, G.: Multi-model simulations of carbon monoxide: Comparison with observations and projected near-future changes, J. Geophys. Res., 111, D19306, doi:10.1029/2006JD007100, 2006.
Spurr, R. and Natraj, V.: A linearized two-stream radiative transfer code for fast approximation of multiplescatter fields, J. Quant. Spectrosc. Ra., 112, 2630-2637, doi:10.1016/j.jqsrt.2011.06.014, 2011.

Stier, P., Feichter, J., Kinne, S., Kloster, S., Vignati, E., Wilson, J., Ganzeveld, L., Tegen, I., Werner, M., Balkanski, Y., Schulz, M., Boucher, O., Minikin, A., and Petzold, A.: The aerosol-climate model ECHAM5-HAM, Atmos. Chem. Phys., 5, 1125-1156, doi:10.5194/acp-5-1125-2005, 2005.

Tikhonov, A.: On the solution of incorrectly stated problems and a method of regularization, Dokl. Acad. Nauk SSSR, 151, 501504, 1963.

Tol, P., Landgraf, J., and Aben, I.: Instrument noise model for the Sentinel 5 SWIR bands, Report, Netherlands Insitute for Space Research, SRON, Utrecht, the Netherlands, 2011.

Ustinov, E. A.: Inverse problem of photometric observation of solar radiation reflected by an optically dense planetary atmosphere. Mathematical methods and weighting functions of linearized inverse problem, Cosmic Res., 29, 519-532, 1991.

van Deelen, R., Hasekamp, O. P., and Landgraf, J.: Accurate modeling of spectral fine-structure in Earth radiance spectra measured with the Global Ozone Monitoring Experiment, Appl. Opt., 46, 243-252, 2007.

van Diedenhoven, B., Hasekamp, O., and Landgraf, J.: Retrieval of cloud parameters from satellite-based reflectance measurements in the ultraviolet and the oxygen A-band, J. Geophys. Res., 112, D15208, doi:10.1029/2006JD008155, 2007.

Veefkind, P.: TROPOMI on the ESA Sentinel-5 Precursor: a GMES mission for Global Observations of the Atmospheric Composition for Climate and Air Quality Applications, Remote Sens. Envirom., 120, 70-83, 2012.

Vidot, J., Landgraf, J., Hasekamp, O., Butz, A., Galli, A., Tol, P., and Aben, I.: Carbon monoxide from shortwave infrared reflectance measurements: A new retrieval approach for clear sky and partially cloudy atmospheres, Remote Sens. Environ., 120, 255-266, 2012.

Walter, H. H., Landgraf, J., and Hasekamp, O. P.: Linearization of a pseudo-spherical vector radiative transfer model, J. Quant. Spectrosc. Ra., 85, 251-283, doi:10.1016/S0022-4073(03)00228-0, 2004.

Walter, H. H., Landgraf, J., Spada, F., and Doicu, A.: Linearization of a radiative transfer model in spherical geometry, J. Geophys. Res., 111, D24304, doi:10.1029/2005JD007014, 2006.

Wassmann, A., Borsdorff, T., aan de Brugh, J. M. J., Hasekamp, O. P., Aben, I., and Landgraf, J.: The direct fitting approach for total ozone column retrievals: a sensitivity study on GOME2/MetOp-A measurements, Atmos. Meas. Tech., 8, 4429-4451, doi:10.5194/amt-8-4429-2015, 2015.

Winker, D. M., Hunt, W. H., and McGill, M. J.: Initial performance assessment of CALIOP, Geophys. Res. Lett., 34, L19803, doi:10.1029/2007GL030135, 2007.

Zdunkowski, W. G., Welch, R. M., and Korb, G.: An Investigation of the Structure of Typical Two-stream-methods for the Calculation of Solar Fluxes and Heating Rates in Clouds, Contrib. Atmos. Phys., 53, 147-166, 1979. 